\title{
Usutu virus escapes langerin-induced restriction to productively infect human Langerhans cells, unlike West Nile virus
}

Marie-France Martin ${ }^{1 \pi}$, Ghizlane Maarifi ${ }^{1 \pi}$, Hervé Abiven ${ }^{1}$, Marine Seffals $^{2}$, Nicolas Mouchet ${ }^{2}$, Cécile Beck $^{3}$, Charles Bodet ${ }^{4}$, Nicolas Lévèque ${ }^{4}$, Nathalie J. Arhel ${ }^{1}$, Fabien P. Blanchet ${ }^{1}$, Yannick Simonin ${ }^{5}$, Sébastien Nisole ${ }^{1^{*}}$

${ }^{1}$ Institut de Recherche en Infectiologie de Montpellier (IRIM), Université de Montpellier, CNRS, Montpellier, France.

${ }^{2}$ Plate-Forme H2P2, Université de Rennes 1, Biosit, Rennes, France.

${ }^{3}$ UMR1161 Virologie, INRAE, ANSES, Ecole Nationale Vétérinaire d'Alfort, Université Paris-Est, Maisons-Alfort, France.

${ }^{4}$ Laboratoire Inflammation, Tissus Epithéliaux et Cytokines, LITEC EA 4331, Université de Poitiers, Poitiers, France.

${ }^{5}$ Pathogenesis and Control of Chronic and Emerging Infections, Université de Montpellier, INSERM, EFS, Montpellier, France.

I These authors contributed equally to this work.

* Corresponding author

E-mail: sebastien.nisole@inserm.fr (SN) 


\begin{abstract}
Usutu virus (USUV) and West Nile virus (WNV) are phylogenetically close emerging arboviruses transmitted by mosquitoes, and constitute a global public health threat. Since USUV and WNV enter the body through the skin, the first immune cells they encounter are skin-resident dendritic cells, the most peripheral outpost of immune defense. This unique network is composed of Langerhans cells (LCs) and dermal DCs, which reside in the epidermis and the dermis, respectively.

Using human skin explants, we show that while both viruses can replicate in keratinocytes, they can also infect resident DCs with distinct tropism, since WNV preferentially infects DCs in the dermis, whereas USUV has a greater propensity to infect LCs. Using both purified human epidermal LCs (eLCs) and monocyte derived LCs (MoLCs), we confirm that LCs sustain a faster and more efficient replication of USUV compared with WNV and that this correlates with a more intense innate immune response to USUV compared with WNV. Next, we show that ectopic expression of the LC-specific C-type lectin receptor (CLR), langerin, in HEK293T cells allows WNV and USUV to bind and enter, but supports the subsequent replication of USUV only. Conversely, blocking or silencing langerin in MoLCs or eLCs made them resistant to USUV infection, thus demonstrating that USUV uses langerin to enter and replicate in LCs. Altogether, our results demonstrate that LCs constitute privileged target cells for USUV in human skin, because langerin favors its entry and replication. Intriguingly, this suggests that USUV efficiently escapes the antiviral functions of langerin, which normally safeguards LCs from most viral infections.
\end{abstract}




\section{Introduction}

Usutu virus (USUV) and West Nile Virus (WNV) are emerging arboviruses belonging to the Flavivirus genus of the Flaviviridae family, which comprise many other human pathogenic viruses, including Zika virus (ZIKV), Dengue virus (DENV) or Yellow fever virus (YFV). They are phylogenetically close and both belong to the Japanese encephalitis virus (JEV) antigenic complex [1]. WNV and USUV have recently expanded outside of Africa, where they both originate: WNV is now endemic throughout much of the world whereas the spread of USUV has recently dramatically increased in Europe and it has become endemic in several European countries [2-6]. Both viruses have caused several outbreaks among humans and birds and are therefore considered as serious potential threats to human and animal health $[3-5,7,8]$.

USUV and WNV are maintained in the environment through an enzootic cycle involving mosquitoes (mainly of the genus Culex) and birds [9]. Infected mosquitoes can incidentally transmit the viruses to mammals, such as human and horses, which are dead-end hosts but can both develop severe neurological disorders. Since viruses are directly inoculated by the bite of an infected mosquito, the first organ to get infected is the skin.

The skin is a complex organ, composed of two main layers: the dermis, made up of connective tissue produced by fibroblasts, and the epidermis, a multilayered stratified epithelium mostly constituted of keratinocytes. The immune surveillance of this most peripheral organ of the body is mainly ensured by a unique network of dendritic cells (DCs), composed of Langerhans cells (LCs), which is the only DC subset that resides in the epidermis, and dermal DCs (dDCs), which are mostly located in the upper dermis $[10,11]$. LCs and dDCs are specialized in the recognition and capture of pathogens in the skin. Following antigen uptake and processing, they migrate to local draining lymph nodes in order to activate effector T cells. The capacity of skin-resident DCs to capture various pathogens is conferred by the expression of specific pattern recognition receptors (PRRs), including C-type lectins receptors (CLRs), which bind carbohydrate structures associated to viruses, fungi or bacteria [12]. Among the CLRs, langerin (or CD207) is exclusively expressed by LCs in humans [13]. Langerin is not only present at the cell surface but also within rod-shaped cytoplasmic organelles with a striated appearance, termed Birbeck granules (BGs) [13]. These are subdomains of the endosomal recycling compartment that are involved in pathogen degradation and antigen processing $[13,14]$. The role of langerin in the capture and degradation of viruses by LCs has been particularly well described in the case of HIV-1 [15,16]. Another important CLR, mostly expressed by DCs, including dDCs, is DC-SIGN (or CD209) [17]. Although CLRs have clear antiviral functions, many viruses are able to hijack these receptors to their advantage. In particular, DC-SIGN has been shown to bind HIV-1 gp120 and promote efficient trans-infection of T cells [18]. DC-SIGN can also be used by many viruses to infect immature DCs, such as Cytomegalovirus (CMV) [19] or Ebola virus (EBOV) [20] but also several flaviviruses, including DENV and WNV [21-23]. The viral hijacking of langerin seems to be much rarer and has only been formally demonstrated for influenza A virus (IAV), 
38 although it was not determined whether LCs constitute target cells [24]. In contrast, some viruses

39 have been shown to infect Langerhans cells in human skin, including DENV [25-27].

The role of skin cells in WNV infection, amplification and spread has been studied both in vitro and in vivo $[6,28,29]$. In particular, it has been shown that infected mosquitoes inject large quantities

42 of virus into the skin, mostly outside blood vessels [30], and that productively infected keratinocytes

43 probably account for virus dissemination [31]. WNV was shown to infect monocyte-derived DCs 44 [22,32-34], but it not known whether it can infect skin-resident DCs. Although LCs were shown to 45 migrate to local lymph nodes following cutaneous infection with WNV, it was not determined whether 46 these were infected or not [35]. Similarly, since USUV has received less attention than WNV, its 47 cellular tropism in the skin has not yet been investigated and its ability to infect skin-resident DCs is 48 entirely unknown.

49 In this study, we evaluated the capacity of USUV and WNV to infect human skin-resident DCs, 50 including LCs. Using both human primary skin-isolated and monocyte-derived LCs (MoLCs), we 51 report that, although WNV is taken up by LCs to some degree, USUV enters and replicates within 52 LCs much more efficiently than WNV. In particular, we show that human LCs support productive 53 infection of USUV and constitute privileged target cells for this virus. The innate immune response 54 triggered by USUV was also much more intense than that by WNV. Finally, we show that while both 55 USUV and WNV can enter cells following their interaction with langerin, only USUV escapes 56 langerin-induced restriction in order to replicate in Langerhans cells. 


\section{Results}

\section{USUV has the propensity to infect human epidermal Langerhans cells (eLCs)}

First of all, we compared the capacity of USUV and WNV to infect DCs within human skin, using a strain of USUV Africa 2 (USUV AF2) isolated in France in 2018, and a clinical strain of WNV belonging to the lineage 1 (WNV L1). We gently scarified the surface of human skin explants to allow the virus to diffuse in all layers, as occurs during a mosquito bite. Explants were incubated with USUV AF2 or WNV L1 at $10^{7}$ tissue culture infectious dose $50 \%$ (TCID50/ml) for $24 \mathrm{~h}$. Paraffinembedded tissues were analyzed by immunofluorescent staining using an anti-CD1a antibody and a pan-flavivirus antibody targeting the envelope protein $E$ of flaviviruses, to label skin-resident DCs and infected cells, respectively (Figure 1A). As for WNV, most USUV-infected cells were epidermal CD1a-negative, thus suggesting that keratinocytes are also the main targets of USUV. However, a substantial proportion of double-positive cells could also be observed, mostly in the case of USUV infection (Figure 1A). We performed a quantification of CD1a-positive infected cells both in the epidermis and dermis, which confirmed that the proportion of double-positive cells was approximately 2-times higher with USUV compared to WNV (Figure 1B). Interestingly, CD1a-positive cells infected by USUV were almost exclusively found in the epidermis, suggesting that USUV preferentially infects LCs. In contrast, WNV was found to infect indifferently epidermal (eLCs) and dermal (dDCs) DCs (Figure 1B). In order to confirm these observations, we infected total epidermal cells or purified eLCs from human skin explants with USUV or WNV for $48 \mathrm{~h}$ at MOI 2 (Figure S1A). As with intact human skin, USUV was found to infect epidermal cells more efficiently than WNV and strikingly, was able to infect nearly all eLCs (Figure 1C). Furthermore, since we labeled the cells with an antibody targeting dsRNA, which recognizes replicating viral genomes, our results suggest that both viruses can replicate within LCs, but that USUV infection is more efficient (Figure 1C). This was confirmed by immunofluorescence staining of purified human epidermal cells, which allowed us to detect USUV replicating within LCs (Figure 1D). Some replicating WNV could be detected, but at very low levels (Figure 1D). Similarly, viral RNA was quantified by RT-qPCR and further confirmed that the amount of USUV in purified epidermal LCs was approximately 10 times higher than of WNV

(Figure 1E). Altogether, our results show that in human skin, both WNV and USUV can infect epidermal CD1a- cells (mainly keratinocytes), as well as eLCs and dDCs. USUV however, was more efficient than WNV to infect skin-resident DCs and in particular eLCs.

\section{USUV replicates at higher rates than WNV in monocyte-derived LCs}

To further investigate the propensity of USUV to infect human LCs, we moved to a model of human monocyte-derived cells. Since monocytes can be differentiated into either DCs (MoDCs) or LCs (MoLCs) (Figure S1B), we first compared the kinetics of USUV and WNV replication in these cells.

92 In order to exclude any strain-specific phenotype, we included 2 more viral lineages in our study: 93 USUV Europe 2 (EU2, TE20421/Italy/2017), a lineage that has been involved in several severe 
clinical cases [36,37], and a strain of WNV L2 (WNV-6125/France/2018), a lineage that actively circulates in Europe since 2004 [38]. We infected monocytes, or autologous MoDCs or MoLCs, and infection was followed over time by RT-qPCR. WNV strains showed slow and low replication rates that were comparable in the 3 cell types, whereas USUV replication was much faster and higher in amplitude in MoLCs and MoDCs, and peaked at 16 and $24 \mathrm{~h}$ post-infection (hpi), respectively (Figure 2A). Interestingly, USUV EU2 was the most virulent strain in these 2 models, in terms of kinetic and replication rates.

In order to confirm that MoLCs are able to support productive infection of USUV and WNV, we titrated the viruses produced by MoLCs over time. As shown in Figure 2B, replicative virus was detected in MoLC culture medium as early as $24 \mathrm{hpi}$ and the highest viral production rate was again found for USUV EU2, which reached $4.10^{7} \mathrm{TCID} 50 / \mathrm{ml}$. Noteworthy, the viral replication kinetics of USUV strains showed multistep growth curves, typical of an effective viral replication (Figure 2B, top), while WNV L1 and WNV L2 RNA copies were significantly much lower and showed no active replication (Figure 2B, bottom). Similarly, immunofluorescence labeling of dsRNA in MoLCs infected with all 4 viral strains in order to evaluate the viral replication showed higher replication levels for USUV strains compared with WNV (Figure 2C). We also looked at Mx1 expression, a type I interferon-induced protein, in order to evaluate the innate immune response of MoLCs to infection [39]. Interestingly, we observed that dsRNA and Mx1 staining were mutually exclusive, a pattern that was particularly pronounced in the case of USUV (Figure 2C), suggesting that MoLCs respond to USUV infection by secreting interferon, thus partially inhibiting viral replication (Figure $2 \mathrm{C}$ ). In accord with this, we found that type I IFN strongly inhibits the replication of all 4 viral strains both in MoDCs and in MoLCs (Figure S2).

\section{USUV induces a higher innate immune response than WNV in MoLCs}

To further investigate the innate immune response of MoLCs to WNV and USUV infection, we first compared the production of type I IFN triggered by WNV and USUV in monocytes, MoDCs and MoLCs. In monocytes, only USUV EU2 infection led to a very limited amount of type I IFN secreted in the culture medium from $48 \mathrm{hpi}$ (Figure 3A). In MoDCs and MoLCs however, high amounts of type I IFN were produced, especially following USUV infection (Figure 3A). The divergence between USUV and WNV infection was particularly dramatic in MoLCs, since WNV triggered very little or no IFN production, whereas USUV strains induced a fast and potent secretion of up to $10^{8} \mathrm{U} / \mathrm{ml}$ at 24 hpi (Figure 3A). Therefore, there is a good correlation between the susceptibility to infection of MoLCs by a given virus (Figure 2A) and their propensity to secrete IFN (Figure 3A). Next, we investigated whether USUV triggers a globally more intense antiviral innate immune response than WNV in MoLCs. To this end, we quantified the expression of a large panel of cytokines, chemokines and interferon-stimulated genes (ISGs) by RT-qPCR in MoLCs at 24 hpi. As anticipated, the induction of all transcripts was higher in USUV- compared to WNV-infected cells and once again, USUV EU2 was the most potent trigger (Figure 3B). A selection of cytokines was also quantified at 
132 the protein level in the culture medium of MoLCs at $48 \mathrm{hpi}$, and confirmed the former observations

133 (Figure 3C). Therefore, our results suggest that the efficient replication of USUV in MoLCs triggers

134 a fast and potent innate immune response.

\section{USUV escapes post-entry langerin-mediated degradation}

137 Since we showed that USUV can infect DCs and has a strong tropism for both MoLCs and epidermal 138 LCs, we sought to decipher whether it has a better propensity to use DC-expressed CLR as entry 139 receptors compared with WNV. To do this, we ectopically expressed human DC-SIGN or langerin in 140 HEK293T cells (Figure S3A), which are poorly permissive to USUV and WNV at low MOI, and 141 evaluated both viral entry and replication. At $48 \mathrm{~h}$ post-infection, the expression of DC-SIGN 142 enhanced WNV infection by more than 6 times, in agreement with previous studies [22,32], whereas 143 the expression of langerin did not allow the entry and/or replication of WNV in HEK293T cells (Figure 144 4A). In contrast, both DC-SIGN and langerin promoted infection by USUV (Figure 4A), thus 145 suggesting that unlike WNV, USUV can also use langerin as a receptor to enter and replicate. These 146 observations were confirmed by quantifying the amount of viral RNA in infected cells by RT-qPCR, 147 since we observed that DC-SIGN expression led to a 10-fold increase of both USUV and WNV 148 infection, whereas langerin expression only increased USUV replication (Figure 4B). We also 149 confirmed by western-blot that langerin expression allowed a marked enhancement of USUV E 150 protein detection in infected cells at $24 \mathrm{~h}$ and $48 \mathrm{~h}$ (Figure $4 \mathrm{C}$ ). In the case of WNV, again, langerin 151 expression had either no effect on virus replication or was even deleterious. These results pointed 152 to two possible interpretations: on the one hand, this could mean that only USUV can use langerin 153 as a receptor, on the other hand, it is possible that both viruses enter via langerin, but only USUV 154 manages to escape langerin-induced degradation and replicate. To distinguish between these two 155 hypotheses, we tested the ability of langerin to interact with USUV and WNV envelope proteins by 156 co-immunoprecipitation. To this end, we incubated langerin-overexpressing HEK293T cells with 157 USUV or WNV at MOI 5 for 30 minutes. Since langerin recognizes mannose-rich glycans present 158 on viral glycoproteins, we performed competition experiments using mannan [15,24]. Our results 159 indicate that langerin can interact with both USUV and WNV envelope proteins and that, as 160 expected, this interaction can be inhibited by mannan (Figure 4D). Consistent with the role of langerin 161 in pathogen recognition and capture, we observed by FACS and microscopy that langerin not only 162 binds USUV and WNV, but also allows their internalization (Figures 4E and 4F). Taken together, our 163 results suggest that langerin is able to recognize and internalize both USUV and WNV, but that only 164 USUV is able to escape langerin-induced degradation in order to replicate.

165 In order to confirm our observations in primary cells, we performed further experiments using MoLCs 166 and epidermal LCs. We confirmed that mannan efficiently prevented the infection of LCs by USUV, 167 as shown by RT-qPCR amplification of the viral genome (Figure 5A) and flow cytometry using the 168 dsRNA antibody (Figure 5B). Moreover, we silenced langerin expression in eLCs purified form 169 human epidermis using a specific siRNA (Figure 5C) and show that this efficiently reduced infection 170 by USUV (Figure 5D), thus formally demonstrating that langerin is an entry receptor for USUV in 
bioRxiv preprint doi: https://doi.org/10.1101/2021.08.17.456611; this version posted November $13,2021$. The copyright holder for this

preprint (which was not certified by peer review) is the author/funder, who has granted bioRxiv a license to display the preprint in perpetuity. It is made available under aCC-BY-NC-ND 4.0 International license.

171 human LCs. As observed in HEK293T cells overexpressing langerin (Figure 4F), we noted that

172 endogenous langerin co-localized with incoming USUV in the very first steps of infection, thus

173 suggesting that langerin is co-internalized with USUV virions in eLCs (Figure 5E). 


\section{Discussion}

Arboviruses such as Dengue, Zika, West Nile, or Usutu viruses represent a global public health threat due to globalization and worldwide spread of mosquito vectors $[5,40]$. Since mosquito-borne viruses are directly inoculated in the epidermis and the dermis during blood meals, the skin constitutes the initial site of viral replication and immune response [28]. LCs and dDCs patrol the epidermis and the dermis, respectively, to sense and capture pathogens. For this purpose, they are equipped with unique receptors, known as CLRs, which bind carbohydrate moieties associated to pathogens. Among them, DC-SIGN and langerin are expressed by DCs and LCs, respectively, and act both as PRRs and antigen-uptake receptors. Therefore, DC-SIGN and langerin constitute key receptors, allowing the interception of a variety of pathogens that enter the organism through the skin $[12,41]$. In the case of viruses, glycans are present on their envelope glycoproteins, which are recognized by CLRs, thus inducing their endocytosis and subsequent degradation. But like many cellular defense mechanisms, DC-SIGN and langerin can be bypassed or even hijacked by some viruses to their advantage. Thus, many viruses can use DC-SIGN as a receptor to propagate, either in cis, via the productive infection of DCs, or in trans, if the receptor facilitates the capture and transmission to other cells $[12,18]$. In agreement with this, we found that both USUV and WNV can use DC-SIGN as a receptor, as already shown for many flaviviruses [21,32].

In contrast to DC-SIGN, hijacking of langerin by viruses seems much rarer, and has so far only been demonstrated for IAV in transfected cell lines [24]. In this manuscript, we show that USUV, but not WNV, can use naturally expressed langerin to infect into LCs. Our results not only show that LCs are permissive to USUV, but also that they support productive viral replication. This is a surprising observation, since LCs are notoriously refractory to most viruses, with only a few exceptions, including HSV-1 [42,43] and DENV [25-27]. Among the viral strains that we tested, USUV EU2 showed the fastest and most efficient replication in both DCs and LCs, and also triggered the most intense innate immune response. Interestingly, this viral strain was previously described as being involved in several clinical cases, and was recently shown to be particularly neurovirulent and lethal in mice $[36,37,44]$.

Flaviviruses encode one, two, or no N-linked glycosylation sites on their envelope proteins (E protein) [23]. In the case of WNV, it was shown that, unlike non-glycosylated viral particles, glycosylated strains can use DC-SIGN to infect DCs [32], thus illustrating the importance of $\mathrm{N}$-glycosylations for flavivirus tropism. WNV and USUV E proteins contain a single $\mathrm{N}$-linked glycosylation site at residue 154, whereas most DENV isolates contain glycosylation sites at residues 153 and $67[45,46]$. The specificity of the interactions between glycoproteins and CLRs is complex and depends both on the type and position of glycans. For example, whereas WNV grown in mammalian cells was shown to preferentially use DC-SIGNL as a receptor [22], the introduction of a glycosylation site at position 67 into WNV E protein conferred the capacity to also use DC-SIGN [47]. From the sequence of its USUV E protein, it can be predicted that USUV has, like WNV, a unique glycosylation at N154 [44], thus explaining why both viruses can interact with langerin. 
212 In the case of USUV, we showed that langerin expression not only allows the virus to bind and enter,

213 but also to replicate, thus suggesting that in this case, langerin could be considered as a receptor.

214 However, further work will be required in order to determine whether langerin acts as a bona fide

215 entry receptor or as a proviral factor facilitating virion attachment and entry. In the case of DENV for

216 instance, it was shown that endocytosis-defective DC-SIGN allows viral entry as efficiently as the

217 wild type protein, thus suggesting that DC-SIGN is likely an attachment factor rather than an entry

218 receptor [48]. In our case however, we showed that blocking or silencing langerin in LCs prevents

219 USUV infection, whereas the ectopic expression of langerin in non-permissive cells promotes virus

220 binding, entry and replication. Thus, it is likely that langerin is necessary and sufficient to allow USUV

221 infection of LCs. Furthermore, we show that, although WNV also binds langerin and is internalized

222 in langerin-expressing cells, this does not allow its replication, presumably because the virus is

223 degraded in Birbeck granules, as demonstrated in the case of HIV-1 [15]. Since USUV and WNV

224 are phylogenetically closely related [1,2], our results suggest that adaptation of USUV to human cells

225 involved bypassing langerin-mediated degradation to infect skin-resident Langerhans cells. Further

226 work will be required to uncover the replication advantage that this brings to USUV, in particular

227 whether it contributes more efficiently to its dissemination.

228 As the most peripheral immune cells in the body, LCs are the most exposed. In this respect, it is

229 rather surprising that so few viruses have been found to infect them. It is possible that langerin is

230 more difficult than DC-SIGN to be hijacked by viruses, especially since it forms Birbeck granules that

231 efficiently degrade viruses [13-15]. Alternatively, this may just reflect the fact that LCs are less

232 studied than other types of dendritic cells. In any case, langerin has been described as a potent

233 antiviral barrier that very few viruses are able to overcome [15,24,25,43,49]. How USUV, once inside

234 LCs, manages to avoid degradation in Birbeck granules to replicate in LCs remains an open 235 question.

236 Finally, our results showed a correlation between the susceptibility of LCs to infection and their ability 237 to respond to this infection. Indeed, USUV strains, which infect LCs more efficiently than WNV, also 238 induce a stronger innate immune response. Similarly, among the two USUV strains tested, EU2, the 239 better and fastest replicating strain, was also a more potent inducer of antiviral cytokines and 240 chemokines, including type I IFN. It is intriguing that this virus can replicate so efficiently while 241 inducing such an intense innate response. Viruses are in a speed race with the IFN response to 242 replicate before the IFN-induced antiviral state is established and our results suggest that USUV 243 EU2 is fit enough to win this race. USUV was previously described to induce a stronger interferon 244 response than WNV in MoDCs [50]. Interestingly, authors also showed that USUV was more 245 sensitive than WNV to the antiviral activity of types I and III IFNs [50]. This latter observation might 246 explain why USUV replicates faster than WNV, in order to overtake cellular defenses by completing 247 their replication cycle before the expression of antiviral ISGs. ISGs interfering with WNV replication 248 are now well characterized [51], but no study has yet been performed on USUV, thus illustrating the 249 fact that research on USUV is still in its infancy. A number of studies, including our own, highlight 
250 important differences between USUV and WNV in terms of virus-cell and virus-host interactions.

251 Since it is therefore impossible to transpose our knowledge of WNV onto USUV, more research is 252 clearly needed in order to anticipate the possible worldwide emergence of this virus and the burden 253 to economy and public health it may pose in the future. 
Material and Methods

Cells

256 C6/36 cells (CRL-1660), Vero (CCL-81) and HEK293T (CRL-11268) were purchased from the American Type Culture Collection (ATCC). STING-37 reporter cells were kindly provided by PierreOlivier Vidalain (CIRI, Lyon, France). All cells were cultured with 10\% fetal bovine serum (Serana), $1 \%$ Penicillin/Streptomycin (Gibco) and maintained in $5 \% \mathrm{CO} 2$ at $37^{\circ} \mathrm{C}$, except $\mathrm{C} 6 / 36$ cells which were grown at $28^{\circ} \mathrm{C}$.

\section{Viruses}

263 USUV Europe 2 (TE20421/Italy/2017) was kindly provided by Giovanni Savini (Istituto Zooprofilattico 264 Sperimentale dell' Abruzzo e del Molise "G. Caporale", Teramo, Italy). USUV Africa 2 (Rhône 2705/France/2015) and WNV lineage 2 (WNV-6125/France/2018) were provided by ANSES (National Agency for Food, Environmental and Occupational Health Safety, France). The lineage 1 clinical strain of WNV was isolated from a human brain during the epidemic that occurred in Tunisia in 1997 and was provided by Isabelle Leparc-Goffart (French National Reference Center on Arboviruses, Marseille, France). The origin and history of viral isolates used in the study are summarized in Table 1. Viral strains were amplified on C6/36 cells. Supernatants were collected 5 days after infection and their titers were determined on Vero cells, using the Spearman-Karber method, and expressed as TCID50/ml [52].

Table 1. Origin and history of isolates used in the study.

\begin{tabular}{|c|c|c|c|c|c|c|c|}
\hline Virus & Lineage & Strain & Origin & Year & Source & $\begin{array}{c}\text { Passage } \\
\text { History }\end{array}$ & $\begin{array}{c}\text { Accession } \\
\text { number }\end{array}$ \\
\hline \multirow{2}{*}{ USUV } & AF2 & Rhône 2705 & France & 2015 & Avian & V1C2 & KX601692 \\
\cline { 2 - 7 } & EU2 & TE2042/Italy/2017 & Italy & 2017 & Avian & V1C2 & TE20421 \\
\hline \multirow{2}{*}{ WNV } & L1 & WN-Tunisia-1997 PaH001 & Tunisia & 1997 & Human & V1 & AF418555 \\
\cline { 2 - 8 } & L2 & WNV-6125/France/2018 & France & 2018 & Avian & V1C2 & MT863560 \\
\hline
\end{tabular}

${ }^{*} \mathrm{~V}$ : passage number in Vero cells ; C: passage number in C6/36 cells

\section{Antibodies and reagents}

280 The primary antibodies used were mouse anti-dsRNA (J2 clone, Scicons), mouse pan-flavivirus 281 (4G2 clone, Novus Biologicals), rabbit anti-MX1 (Thermo Fischer Scientific), mouse APC conjugated 282 anti-langerin (clone 10E2, BioLegend), mouse anti-langerin (clone D9H7R, Cell Signaling), 283 recombinant anti-DC-SIGN (clone REA617, Miltenyi Biotec), mouse anti-CD1a (Novus Biologicals), 284 FITC-conjugated anti-CD1a (clone HI149, Miltenyi Biotec), FITC-conjugated anti-HLA-DR (clone 285 AC122, Miltenyi Biotec), mouse anti-HSP90 (clone F-8, Santa Cruz), and mouse anti-GAPDH (clone 6C5, Merck Millipore). Secondary antibodies were goat anti-mouse AF488 (Thermo Fisher 
Scientific), donkey anti-mouse AF568 (Thermo Fisher Scientific), donkey anti-rabbit AF647 (Thermo Fisher Scientific) and goat anti-mouse or anti-rabbit HRP conjugates (GE Healthcare).

Mannan from Saccharomyces cerevisiae was purchased from Sigma-Aldrich.

\section{Plasmids and transfections}

All ectopic transfections were performed using FuGENE ${ }^{\circledR} 6$ Transfection Reagent (Promega) according to the manufacturer's instructions. Plasmids expressing human langerin and DC-SIGN have been described previously [15,53]. Expression of langerin and DC-SIGN constructs was assessed by flow cytometry, western blot, or indirect immunofluorescence. For siRNA transfection, non-targeting control siRNA (siRNA CTR) and siRNA specific for langerin (siRNA langerin) were purchased from Dharmacon as SMARTpools. Transfections were performed using DharmaFECT 4

298 (Dharmacon), as previously described [54].

\section{Blood samples, isolation and culture of human primary cells}

301 Buffy coats from healthy donors were obtained from the Etablissement Français du Sang (EFS, Montpellier, France). PBMCs were isolated by density centrifugation using Lymphoprep medium (STEMCELL Technologies). CD14+ monocytes were isolated from PBMCs using CD14 MicroBeads (Miltenyi Biotec) and subsequently differentiated into MoDCs or MoLCs. Briefly, MoDCs were generated by incubating purified monocytes in Iscove's Modified Dulbecco's Medium (IMDM) 306 supplemented with 10\% FBS, 1\% P/S, 2 mM L-glutamine, 10 mM Hepes, 1\% non-essential aminoacids, $1 \mathrm{mM}$ sodium pyruvate and cytokines GM-CSF (Granulocyte-Macrophage Colony Stimulating Factor, $500 \mathrm{IU} / \mathrm{ml})$ and IL-4 (500 IU/ml), both from Miltenyi Biotec (Cytobox Mo-DC). MoLCs were generated as MoDCs, except for the addition of $10 \mathrm{ng} / \mathrm{ml}$ of TGF- $\beta$ (Peprotech) within the differentiation medium. Immature MoDCs and MoLCs were harvested at day 6 and cell differentiation was estimated by measuring the expression of DC-SIGN, langerin, CD1a and HLADR (class II) by flow cytometry (Figure S1B).

\section{Isolation of epidermal LCs from human skin explants}

315 After the provision of fully informed consent, skin samples were obtained from patients undergoing 316 abdominoplasty or mammoplasty plastic surgery at the Poitiers University Hospital, France. The use 317 of all human skin samples for research studies was approved by the Ethics Committee (committee 318 for the protection of persons) Ouest III (project identification code: DC-2014-2109).

319 Human skin samples processing was adapted from the method previously described in [54]. Briefly, 320 skin sheets were cut into $1 \mathrm{~cm}^{2}$ pieces and incubated with agitation in shaking water bath (at 175 321 strokes/minute) in RPMI medium (Gibco) containing collagenase A ( $1 \mathrm{mg} / \mathrm{ml}$, Roche), DNase I (20 $322 \mathrm{U} / \mathrm{ml}$, Sigma, D4263-5VL) and Dispase II (1 mg/ml, Roche) overnight at $37^{\circ} \mathrm{C}$, after which the 323 epidermis was mechanically separated from the dermis using forceps. Epidermal sheets were 324 cultured separately in RPMI with 10\% human AB serum (Thermo Fisher Scientific) and 1\% 325 Penicillin/Streptomycin/Amphotericin B solution for $48 \mathrm{~h}$, after which migratory cells were collected 
326 from media. Migratory cells were either used directly for experiments or subjected to a positive 327 selection using CD1a Microbeads (Miltenyi Biotec), in order to enrich epidermal Langerhans cells.

328 The expression of CD207 and HLA-DR (class II) was assessed by flow cytometry. Staining was 329 performed on both total epidermal cells and following purification of CD1a+ cells, in order to estimate 330 the enrichment rate (Figure S1A).

\section{Infection of human skin explants and multiplex immunofluorescence assay}

333 The outer layer of $1 \mathrm{~cm}^{2}$ human skin explants was gently scarified using a fine needle and the viral 334 inoculum was applied to the scarified surface, in order to allow viruses to spread inside the tissue. 335 The pieces of skin were infected with $10^{7} \mathrm{TCID} 50 / \mathrm{ml}$ of USUV AF2 or WNV L1 in $500 \mu \mathrm{L}$ of RPMI 336 containing $2 \%$ human $A B$ serum and $1 \%$ of $P / S$. After $4 \mathrm{~h}$ at $37^{\circ} \mathrm{C}$, medium was complemented to $33710 \%$ of human $A B$ serum. At $24 \mathrm{~h}$ post-infection, skin pieces were fixed in $4 \%$ paraformaldehyde 338 (PFA) for $24 \mathrm{~h}$ at $4^{\circ} \mathrm{C}$, and dehydrated in $70^{\circ}$ ethanol. Samples were sent to the H2P2 platform 339 (University of Rennes, France) to perform multiplex immunofluorescence assays. Paraffin340 embedded tissue was cut at $4 \mu \mathrm{m}$, mounted on Adhesion slides (TOMO) and dried at $58^{\circ} \mathrm{C}$ for $12 \mathrm{~h}$. 341 Immunofluorescent staining was performed on the Discovery ultra-Automated IHC stainer, using the 342 Discovery Rhodamine kit and the Discovery FAM kit (Ventana Medical Systems). Following 343 deparaffination with Ventana Discovery Wash solution (Ventana Medical Systems) at $75{ }^{\circ} \mathrm{C}$ for 8 344 min, antigen retrieval was performed by using Tris-based buffer solution CC1 (Ventana Medical 345 Systems) at $95^{\circ} \mathrm{C}$ to $100^{\circ} \mathrm{C}$ for $40 \mathrm{~min}$. Endogen peroxidase was blocked with Disc inhibitor (Ventana 346 Medical Systems) for $8 \mathrm{~min}$ at $37^{\circ} \mathrm{C}$. After rinsing with reaction buffer (Ventana Medical Systems), 347 slides were incubated at $37^{\circ} \mathrm{C}$ for $60 \mathrm{~min}$ with the pan-flavivirus antibody. After rinsing, signal 348 enhancement was performed using anti-mouse HRP antibody (Ventana Medical Systems) incubated 349 for $16 \mathrm{~min}$ and the discovery Rhodamine kit (Ventana Medical Systems). Following denaturation with 350 Ventana solution CC2 (Ventana Medical Systems) at $100^{\circ} \mathrm{C}$ for $8 \mathrm{~min}$, slides were incubated at $37^{\circ} \mathrm{C}$ 351 for 60 min with the anti-CD1a antibody. After rinsing, signal enhancement was performed using anti352 mouse HRP antibody (Ventana Medical Systems) incubated for 16 min and the discovery FAM kit 353 (Ventana Medical Systems). Slides were then counterstained for 4 min with DAPI and rinsed. After 354 removal from the instrument, slides were manually rinsed and placed on coverslips. Images were 355 acquired on a confocal scanner. Quantifications were performed with the image analysis platform 356 Halo (V3.2.1851.371), using the HighPlex module (V4.04). Whole sections were analyzed 357 (representing approximately 100,000 cells per condition). The number of cells positive for CD1a and pan-flavivirus staining was determined in the epidermis and in the dermis.

\section{Quantification of secreted cytokines and chemokines}

361 Total IFN secreted by monocytes, MoDCs and MoLCs was titrated on STING-37 reporter cells, which correspond to HEK293 cells stably expressing an IFN-stimulated response element (ISRE)-

363 luciferase reporter gene [55]. A standard curve was established by applying known titers of 364 recombinant IFN- $\alpha 2 a$ (R\&D Systems) onto STING-37 cells. Luciferase induction in STING-37 cells 
was determined using the Bright-Glo reagent (Promega), according to manufacturer's instructions, and luminescence signal was acquired on a TECAN Infinite 200. Quantification of cytokine and chemokine levels in culture media was performed using the LEGENDPlex kit from BioLegend (human anti-virus response panel), according to the manufacturer's recommendations.

\section{Immunofluorescence assays}

371 Cells were plated on poly-D-lysine coated coverslips, fixed with 4\% PFA (Alfa Aesar) for 10 min, permeabilized with $0.1 \%$ Triton $\times 100$ for $15 \mathrm{~min}$, neutralized with $50 \mathrm{mM} \mathrm{NH}_{4} \mathrm{Cl}$ for $10 \mathrm{~min}$, and blocked with $2 \%$ BSA for $10 \mathrm{~min}$. Cells were incubated with primary and secondary antibodies for 1 $\mathrm{h}$ and $45 \mathrm{~min}$, respectively, at room temperature in a wet chamber. Finally, cells were labelled with Hoescht and mounted in SlowFade antifade reagent (Thermo Fischer Scientific). Images were acquired with a Leica SP5-SMD confocal microscope. Mander's coefficients were determined by counting 3 fields of around 300 cells per condition using the JAcoP plugin (ImageJ).

\section{Flow cytometry analysis}

380 All cells were fixed with $2 \%$ PFA for $30 \mathrm{~min}$ and permeabilized in a PBS $/ 1 \%$ BSA $/ 0.05 \%$ saponin solution for 30 min prior to intracellular staining with corresponding primary antibodies for $1 \mathrm{~h}$ at $4^{\circ} \mathrm{C}$ diluted in the permeabilization solution and then incubated with the corresponding secondary antibody for $30 \mathrm{~min}$. For flow cytometry analysis, all acquisitions were done with Fortessa cytometer

384 (B Becton Dickinson D), data were collected with FACSDiva software (Becton Dickinson) and were processed with FlowJo software (Treestar Inc.).

\section{Immunoprecipitation}

HEK293T cells were transfected with an empty plasmid (pcDNA3.1) or with a plasmid encoding langerin and infected $24 \mathrm{~h}$ post-transfection with USUV AF2 and WNV L1 at MOI 5.30 minutes after infection, cells were lysed in IP lysis buffer (50 mM Tris-HCl (pH7.4), $150 \mathrm{mM} \mathrm{NaCl} 1 \mathrm{mM}$ EDTA, 1\% Triton X-100, $20 \mathrm{mM} \mathrm{N}$-ethylmaleimide, EDTA-free protease inhibitor cocktail (Roche)) for $1 \mathrm{~h}$ at $4^{\circ} \mathrm{C}$. Cell lysates were then incubated overnight at $4^{\circ} \mathrm{C}$ with pan-flavivirus anti-E antibody and protein G Sepharose beads (Thermo Fisher Scientific). Beads were washed three times and eluted with $2 \times$ SDS loading buffer before Western blot analysis.

\section{Western Blot}

For the detection of the viral envelope protein, the anti-pan-flavivirus antibody was used under nonreducing and non-denaturing conditions. Briefly, cells were lysed in buffer containing Tris $\mathrm{pH} 7.6$ $1 \mathrm{mM}, \mathrm{NaCl} 150 \mathrm{mM}$, Deoxycholate $0.1 \%$ (deoxycholic acid), EDTA $1 \mathrm{mM}$ and Triton $1 \%$ during 30 min at $4^{\circ} \mathrm{C}$ and then Laemmli $2 \mathrm{X}$ with SDS but without $\beta$-mercaptoethanol. Cell lysates were loaded on 10\% ProSieve gel (LONZA, LON50618), then subjected to electrophoresis. Chemiluminescent

402 acquisitions were done on a Chemidoc ${ }^{\mathrm{TM}}$ MP Imager and analyzed using Image Lab ${ }^{\mathrm{TM}}$ desktop software (Bio-Rad Laboratories). 
bioRxiv preprint doi: https://doi.org/10.1101/2021.08.17.456611; this version posted November 13, 2021. The copyright holder for this preprint (which was not certified by peer review) is the author/funder, who has granted bioRxiv a license to display the preprint in perpetuity. It is made available under aCC-BY-NC-ND 4.0 International license.

404

405

406

407

408

409

410

411

412

413

414

415

416

\section{RT-qPCR}

Total RNA was extracted using a RNeasy Mini kit (Qiagen) following the manufacturer's instructions. RNA concentration and purity were evaluated by spectrophotometry (NanoDrop 2000c, Thermo Fischer Scientific). A maximum of 500 ng of RNA were reverse transcribed with both oligo dT and random primers using a PrimeScript RT Reagent Kit (Perfect Real Time, Takara Bio Inc.) in a $10 \mu \mathrm{L}$ reaction. Real-time PCR reactions were performed in duplicate using Takyon ROX SYBR MasterMix blue dTTP (Eurogentec) on an Applied Biosystems QuantStudio 5 (Thermo Fischer Scientific). Transcripts were quantified using the following program: $3 \mathrm{~min}$ at $95^{\circ} \mathrm{C}$ followed by 40 cycles of 15 $\mathrm{s}$ at $95^{\circ} \mathrm{C}, 20 \mathrm{~s}$ at $60^{\circ} \mathrm{C}$ and $20 \mathrm{~s}$ at $72^{\circ} \mathrm{C}$. Values for each transcript were normalized to expression levels of RPL13A (60S ribosomal protein L13a), using the 2- $\Delta \Delta \mathrm{Ct}$ method. Primers used for quantification of transcripts are indicated within Table 2.

\section{Table 2. Primers used in RT-qPCR analyses}

\begin{tabular}{|c|c|c|}
\hline Target & Forward primer $\left(5^{\prime}->3^{\prime}\right)$ & Reverse primer $\left(5^{\prime}->3^{\prime}\right)$ \\
\hline RPL13A & AACAGCTCATGAGGCTACGG & TGGGTCTTGAGGACCTCTGT \\
\hline USUV & AACAGACGGTGATGCGAACT & TACAGCTTCGGAAACGGCTT \\
\hline WNV & AGTTGAGTAGACGGTGCTGC & CTCCTTCCGAGACGGTTCTG \\
\hline IFN- $\alpha 2$ & CTTGACTTGCAGCTGAGCAC & GCTCACCCATTTCAACCAGT \\
\hline IFN- $\boldsymbol{\beta}$ & TGCTCTCCTGTTGTGCTTCTC & CAAGCCTCCCATTCAATTGCC \\
\hline IFN-Y & GGCAGCCAACCTAAGCAAGAT & CAGGGTCACCTGACACATTCA \\
\hline IFN- $\lambda 1$ & TTCCAAGCCCACCACAACTG & GTGACTCTTCCAAGGCGTCC \\
\hline IFN- $\lambda 2 / 3$ & CTGCCACATAGCCCAGTTCA & TCCTTCAGCAGAAGCGACTC \\
\hline IRF7 & CAGATCCAGTCCCAACCAAG & GTCTCTACTGCCCACCCGTA \\
\hline DDX60 & AACAGGATGAATGAAGGAGATGCT & AGCTCACGCAAGGAAACACT \\
\hline OASL & TCGTGAAACATCGGCCAACT & AAGAGCATAGAGAGGGGGCA \\
\hline PKR & GTGGACCTCTACGCTTTGGG & GATGCCATCCCGTAGGTCTG \\
\hline RIG-I & ATCCAAACCAGAGGCAGAGGAA & ACTGCTTCGTCCCATGTCTGAA \\
\hline TRIM25 & CTTACCCAGCAAGCTTCCCA & GCACCTTGGCCTTGAGAGAT \\
\hline IFITM1 & AGGAAGATGGTTGGCGACG & GCCGAATACCAGTAACAGGATGA \\
\hline IFITM2 & TTGTGCAAACCTTCTCTCCTGT & CCCAGCATAGCCACTTCCTG \\
\hline IFITM3 & GAAGATGGTTGGCGACGTGA & CACTGGGATGACGATGAGCA \\
\hline MX1 & AAGCTGATCCGCCTCCACTT & TGCAATGCACCCCTGTATACC \\
\hline MX2 & CTGGCCAGGTGGAGAAAGAG & TCAGGGGAGGTGATCTCCAG \\
\hline IFIT1 & ATGCGATCTCTGCCTATCGC & CCTGCCTTAGGGGAAGCAAA \\
\hline IFIT2 & AATAGGACACGCTGTGGCTC & AGGCTGGCAAGAATGGAACA \\
\hline IFIT3 & AACAGATGTCCTCCGCAGTG & TGTGGATTCCAACACCCGTT \\
\hline IFI27 & ATCAGCAGTGACCAGTGTGG & GGCCACAACTCCTCCAATCA \\
\hline ISG15 & CAGCGAACTCATCTTTGCCAG & GACACCTGGAATTCGTTGCC \\
\hline ISG20 & GAGCGCCTCCTACACAAGAG & TAGAGCTCCATCGTTGCCCT \\
\hline IL-1及 & GGCATCCAGCTACGAATCTC & GAACCAGCATCTTCCTCAGC \\
\hline IL-4 & AACAGCCTCACAGAGCAGAAGAC & GCCCTGCAGAAGGTTTCCTT \\
\hline IL-6 & TAACCACCCCTGACCCAACC & ATTTGCCGAAGAGCCCTCAG \\
\hline IL-12a & TCAGCAACATGCTCCAGAAG & GGTAAACAGGCCTCCACTGT \\
\hline IL-12b & GGACATCATCAAACCTGACC & AGGGAGAAGTAGGAATGTGG \\
\hline IL-17a & AAGAACTTCCCCCGGACTGT & AGGTGAGGTGGATCGGTTGT \\
\hline IL-17f & TCACGTAACATCGAGAGCCG & GCAGCCCAAGTTCCTACACT \\
\hline IL-22 & CACCTTCATGCTGGCTAAGGA & TCATCAGATAGCAGCGCTCAC \\
\hline IL-23a & CCCAAGGACTCAGGGACAAC & AGAGAAGGCTCCCCTGTGAA \\
\hline TNF- $\alpha$ & GGCGTGGAGCTGAGAGATAAC & GGTGTGGGTGAGGAGCACAT \\
\hline CXCL1 & CGGAAAGCTTGCCTCAATCCT & CAGTTGGATTTGTCACTGTTCAGC \\
\hline CCL3 & CCAGTTCTCTGCATCACTTGCT & TGGCTGCTCGTCTCAAAGTAG \\
\hline CCL5 & CTGCTTTGCCTACATTGCCC & TCGGGTGACAAAGACGACTG \\
\hline CXCL9 & CCAACCAAGGGACTATCCACCT & GGCTGACCTGTTTCTCCCACTT \\
\hline CXCL10 & CGCTGTACCTGCATCAGCAT & GCAATGATCTCAACACGTGGAC \\
\hline
\end{tabular}




\section{Acknowledgments}

418 We acknowledge the imaging facility MRI (Montpellier, France), member of the national 419 infrastructure France-Biolmaging, supported by the French National Research Agency (ANR-10420 INBS-04, "Investissements d'avenir" program). We also thank Dr Alain Fautrel from the H2P2 421 platform (Université de Rennes 1, Rennes, France) for his help and his reactivity.

422 This work was supported by the Labex EpiGenMed, an "Investissements d'Avenir" program (ANR423 10-LABX-12-01). M.F.M was the recipient of a doctoral fellowship from the Labex EpiGenMed. G.M. 424 was supported by a grant from the Agence National de la Recherche sur le SIDA et les Hépatites 425 virales (ANRS).

\section{Declaration of interests}

428 The authors declare no competing interests. 


\section{References}

1. Poidinger M, Hall RA, Mackenzie JS. Molecular characterization of the Japanese encephalitis serocomplex of the flavivirus genus. Virology. 1996;218: 417-421.

doi:10.1006/viro.1996.0213

2. Weissenböck H, Kolodziejek J, Url A, Lussy H, Rebel-Bauder B, Nowotny N. Emergence of Usutu virus, an African mosquito-borne flavivirus of the Japanese encephalitis virus group, central Europe. Emerg Infect Dis. 2002;8: 652-656. doi:10.3201/eid0807.020094

3. Clé M, Beck C, Salinas S, Lecollinet S, Gutierrez S, Van de Perre P, et al. Usutu virus: A new threat? Epidemiol Infect. 2019;147: e232. doi:10.1017/S0950268819001213

4. Roesch F, Fajardo A, Moratorio G, Vignuzzi M. Usutu Virus: An Arbovirus on the Rise. Viruses. 2019;11. doi:10.3390/v11070640

5. Pierson TC, Diamond MS. The continued threat of emerging flaviviruses. Nat Microbiol. 2020;5: 796-812. doi:10.1038/s41564-020-0714-0

6. Suthar MS, Diamond MS, Gale M. West Nile virus infection and immunity. Nat Rev Microbiol. 2013;11: 115-128. doi:10.1038/nrmicro2950

7. Gill CM, Kapadia RK, Beckham JD, Piquet AL, Tyler KL, Pastula DM. Usutu virus disease: a potential problem for North America? J Neurovirol. 2020;26: 149-154. doi:10.1007/s13365-01900818-y

8. Nisole S. [Usutu, that which does not kill us, makes us stronger]. Virologie (Montrouge). 2018;22: 231-232. doi:10.1684/vir.2018.0750

9. Pfeffer M, Dobler G. Emergence of zoonotic arboviruses by animal trade and migration. Parasit Vectors. 2010;3: 35. doi:10.1186/1756-3305-3-35

10. Kashem SW, Haniffa M, Kaplan DH. Antigen-Presenting Cells in the Skin. Annu Rev Immunol. 2017;35: 469-499. doi:10.1146/annurev-immunol-051116-052215

11. Malissen B, Tamoutounour S, Henri S. The origins and functions of dendritic cells and macrophages in the skin. Nat Rev Immunol. 2014;14: 417-428. doi:10.1038/nri3683

12. Bermejo-Jambrina M, Eder J, Helgers LC, Hertoghs N, Nijmeijer BM, Stunnenberg M, et al. C-Type Lectin Receptors in Antiviral Immunity and Viral Escape. Front Immunol. 2018;9. doi:10.3389/fimmu.2018.00590

13. Valladeau J, Ravel O, Dezutter-Dambuyant C, Moore K, Kleijmeer M, Liu Y, et al. Langerin, a novel C-type lectin specific to Langerhans cells, is an endocytic receptor that induces the formation of Birbeck granules. Immunity. 2000;12: 71-81. doi:10.1016/s1074-7613(00)80160-0 14. Mc Dermott R, Ziylan U, Spehner D, Bausinger H, Lipsker D, Mommaas M, et al. Birbeck granules are subdomains of endosomal recycling compartment in human epidermal Langerhans cells, which form where Langerin accumulates. Mol Biol Cell. 2002;13: 317-335.

doi:10.1091/mbc.01-06-0300

15. de Witte L, Nabatov A, Pion M, Fluitsma D, de Jong MAWP, de Gruij1 T, et al. Langerin is a natural barrier to HIV-1 transmission by Langerhans cells. Nat Med. 2007;13: 367-371.

doi: $10.1038 / \mathrm{nm} 1541$

16. Ribeiro CMS, Sarrami-Forooshani R, Setiawan LC, Zijlstra-Willems EM, van Hamme JL, Tigchelaar W, et al. Receptor usage dictates HIV-1 restriction by human TRIM5 $\alpha$ in dendritic cell subsets. Nature. 2016;540: 448-452. doi:10.1038/nature20567

17. Geijtenbeek TB, Torensma R, van Vliet SJ, van Duijnhoven GC, Adema GJ, van Kooyk Y, et al. Identification of DC-SIGN, a novel dendritic cell-specific ICAM-3 receptor that supports primary immune responses. Cell. 2000;100: 575-585. doi:10.1016/s0092-8674(00)80693-5

18. Geijtenbeek TBH, Kwon DS, Torensma R, Vliet SJ van, Duijnhoven GCF van, Middel J, et al. DC-SIGN, a Dendritic Cell-Specific HIV-1-Binding Protein that Enhances trans-Infection of T Cells. Cell. 2000;100: 587-597. doi:10.1016/S0092-8674(00)80694-7

19. Halary F, Amara A, Lortat-Jacob H, Messerle M, Delaunay T, Houlès C, et al. Human cytomegalovirus binding to DC-SIGN is required for dendritic cell infection and target cell transinfection. Immunity. 2002;17: 653-664. doi:10.1016/s1074-7613(02)00447-8

20. Alvarez CP, Lasala F, Carrillo J, Muñiz O, Corbí AL, Delgado R. C-type lectins DC-SIGN 
and L-SIGN mediate cellular entry by Ebola virus in cis and in trans. J Virol. 2002;76: 6841-6844. doi:10.1128/jvi.76.13.6841-6844.2002

21. Tassaneetrithep B, Burgess TH, Granelli-Piperno A, Trumpfheller C, Finke J, Sun W, et al. DC-SIGN (CD209) mediates dengue virus infection of human dendritic cells. J Exp Med. 2003;197: 823-829. doi:10.1084/jem.20021840

22. Davis CW, Nguyen H-Y, Hanna SL, Sánchez MD, Doms RW, Pierson TC. West Nile virus discriminates between DC-SIGN and DC-SIGNR for cellular attachment and infection. J Virol. 2006;80: 1290-1301. doi:10.1128/JVI.80.3.1290-1301.2006

23. Carbaugh DL, Lazear HM. Flavivirus Envelope Protein Glycosylation: Impacts on Viral Infection and Pathogenesis. J Virol. 2020;94: e00104-20. doi:10.1128/JVI.00104-20

24. Ng WC, Londrigan SL, Nasr N, Cunningham AL, Turville S, Brooks AG, et al. The C-type Lectin Langerin Functions as a Receptor for Attachment and Infectious Entry of Influenza A Virus. J Virol. 2016;90: 206-221. doi:10.1128/JVI.01447-15

25. Wu SJ, Grouard-Vogel G, Sun W, Mascola JR, Brachtel E, Putvatana R, et al. Human skin Langerhans cells are targets of dengue virus infection. Nat Med. 2000;6: 816-820.

doi:10.1038/77553

26. Duangkhae P, Erdos G, Ryman KD, Watkins SC, Falo LD, Marques ETA, et al. Interplay between Keratinocytes and Myeloid Cells Drives Dengue Virus Spread in Human Skin. Journal of Investigative Dermatology. 2018;138: 618-626. doi:10.1016/j.jid.2017.10.018

27. Cerny D, Haniffa M, Shin A, Bigliardi P, Tan BK, Lee B, et al. Selective susceptibility of human skin antigen presenting cells to productive dengue virus infection. PLoS Pathog. 2014;10: e1004548. doi:10.1371/journal.ppat.1004548

28. Garcia M, Wehbe M, Lévêque N, Bodet C. Skin innate immune response to flaviviral infection. Eur Cytokine Netw. 2017;28: 41-51. doi:10.1684/ecn.2017.0394

29. Briant L, Desprès P, Choumet V, Missé D. Role of skin immune cells on the host susceptibility to mosquito-borne viruses. Virology. 2014;464-465: 26-32.

doi:10.1016/j.virol.2014.06.023

30. Styer LM, Kent KA, Albright RG, Bennett CJ, Kramer LD, Bernard KA. Mosquitoes inoculate high doses of West Nile virus as they probe and feed on live hosts. PLoS Pathog. 2007;3: 1262-1270. doi:10.1371/journal.ppat.0030132

31. Lim P-Y, Behr MJ, Chadwick CM, Shi P-Y, Bernard KA. Keratinocytes are cell targets of West Nile virus in vivo. J Virol. 2011;85: 5197-5201. doi:10.1128/JVI.02692-10

32. Martina BEE, Koraka P, van den Doel P, Rimmelzwaan GF, Haagmans BL, Osterhaus ADME. DC-SIGN enhances infection of cells with glycosylated West Nile virus in vitro and virus replication in human dendritic cells induces production of IFN- $\alpha$ and TNF- $\alpha$. Virus Research. 2008;135: 64-71. doi:10.1016/j.virusres.2008.02.008

33. Zimmerman MG, Bowen JR, McDonald CE, Pulendran B, Suthar MS. West Nile Virus Infection Blocks Inflammatory Response and T Cell Costimulatory Capacity of Human MonocyteDerived Dendritic Cells. J Virol. 2019;93: e00664-19. doi:10.1128/JVI.00664-19

34. Rawle DJ, Setoh YX, Edmonds JH, Khromykh AA. Comparison of attenuated and virulent West Nile virus strains in human monocyte-derived dendritic cells as a model of initial human infection. Virol J. 2015;12: 46. doi:10.1186/s12985-015-0279-3

35. Johnston LJ, Halliday GM, King NJ. Langerhans cells migrate to local lymph nodes following cutaneous infection with an arbovirus. J Invest Dermatol. 2000;114: 560-568. doi:10.1046/j.1523-1747.2000.00904.x

36. Cavrini F, Gaibani P, Longo G, Pierro AM, Rossini G, Bonilauri P, et al. Usutu virus infection in a patient who underwent orthotropic liver transplantation, Italy, August-September 2009. Eurosurveillance. 2009;14: 19448. doi:10.2807/ese.14.50.19448-en

37. Pacenti M, Sinigaglia A, Martello T, De Rui ME, Franchin E, Pagni S, et al. Clinical and virological findings in patients with Usutu virus infection, northern Italy, 2018. Euro Surveill. 2019;24. doi:10.2807/1560-7917.ES.2019.24.47.1900180

38. Hernández-Triana LM, Jeffries CL, Mansfield KL, Carnell G, Fooks AR, Johnson N. Emergence of west nile virus lineage 2 in europe: a review on the introduction and spread of a 
mosquito-borne disease. Front Public Health. 2014;2: 271. doi:10.3389/fpubh.2014.00271

39. Haller O, Staeheli P, Schwemmle M, Kochs G. Mx GTPases: dynamin-like antiviral machines of innate immunity. Trends Microbiol. 2015;23: 154-163. doi:10.1016/j.tim.2014.12.003 40. Girard M, Nelson CB, Picot V, Gubler DJ. Arboviruses: A global public health threat. Vaccine. 2020;38: 3989-3994. doi:10.1016/j.vaccine.2020.04.011

41. Kawamura T, Ogawa Y, Aoki R, Shimada S. Innate and intrinsic antiviral immunity in skin. J Dermatol Sci. 2014;75: 159-166. doi:10.1016/j.jdermsci.2014.05.004

42. Bertram KM, Truong NR, Smith JB, Kim M, Sandgren KJ, Feng KL, et al. Herpes Simplex Virus type 1 infects Langerhans cells and the novel epidermal dendritic cell, Epi-cDC2s, via different entry pathways. PLoS Pathog. 2021;17: e1009536. doi:10.1371/journal.ppat.1009536 43. Puttur FK, Fernandez MA, White R, Roediger B, Cunningham AL, Weninger W, et al. Herpes simplex virus infects skin gamma delta $T$ cells before Langerhans cells and impedes migration of infected Langerhans cells by inducing apoptosis and blocking E-cadherin downregulation. J Immunol. 2010;185: 477-487. doi:10.4049/jimmunol.0904106

44. Clé M, Constant O, Barthelemy J, Desmetz C, Martin MF, Lapeyre L, et al. Differential neurovirulence of Usutu virus lineages in mice and neuronal cells. J Neuroinflammation. 2021;18: 11. doi:10.1186/s12974-020-02060-4

45. Mukhopadhyay S, Kim B-S, Chipman PR, Rossmann MG, Kuhn RJ. Structure of West Nile Virus. Science. 2003;302: 248-248. doi:10.1126/science.1089316

46. Kuhn RJ, Zhang W, Rossmann MG, Pletnev SV, Corver J, Lenches E, et al. Structure of Dengue Virus: Implications for Flavivirus Organization, Maturation, and Fusion. Cell. 2002;108: 717-725. doi:10.1016/S0092-8674(02)00660-8

47. Davis CW, Mattei LM, Nguyen H-Y, Ansarah-Sobrinho C, Doms RW, Pierson TC. The Location of Asparagine-linked Glycans on West Nile Virions Controls Their Interactions with CD209 (Dendritic Cell-specific ICAM-3 Grabbing Nonintegrin)*. Journal of Biological Chemistry. 2006;281: 37183-37194. doi:10.1074/jbc.M605429200

48. Lozach P-Y, Burleigh L, Staropoli I, Navarro-Sanchez E, Harriague J, Virelizier J-L, et al. Dendritic cell-specific intercellular adhesion molecule 3-grabbing non-integrin (DC-SIGN)mediated enhancement of dengue virus infection is independent of DC-SIGN internalization signals. J Biol Chem. 2005;280: 23698-23708. doi:10.1074/jbc.M504337200

49. Maarifi G, Czubala MA, Lagisquet J, Ivory MO, Fuchs K, Papin L, et al. Langerin (CD207) represents a novel interferon-stimulated gene in Langerhans cells. Cell Mol Immunol. 2020;17: 547-549. doi:10.1038/s41423-019-0302-5

50. Cacciotti G, Caputo B, Selvaggi C, la Sala A, Vitiello L, Diallo D, et al. Variation in interferon sensitivity and induction between Usutu and West Nile (lineages 1 and 2) viruses. Virology. 2015;485: 189-198. doi:10.1016/j.virol.2015.07.015

51. Martin M-F, Nisole S. West Nile Virus Restriction in Mosquito and Human Cells: A Virus under Confinement. Vaccines (Basel). 2020;8. doi:10.3390/vaccines8020256

52. Kärber G. Beitrag zur kollektiven Behandlung pharmakologischer Reihenversuche. Archiv f experiment Pathol u Pharmakol. 1931;162: 480-483. doi:10.1007/BF01863914

53. Arrighi J-F, Pion M, Wiznerowicz M, Geijtenbeek TB, Garcia E, Abraham S, et al. Lentivirus-mediated RNA interference of DC-SIGN expression inhibits human immunodeficiency virus transmission from dendritic cells to T cells. J Virol. 2004;78: 10848-10855. doi:10.1128/JVI.78.20.10848-10855.2004

54. Maarifi G, Lagisquet J, Hertel Q, Bonaventure B, Chamontin C, Fuchs K, et al. Alarmin S100A9 restricts retroviral infection by limiting reverse transcription in human dendritic cells. EMBO J. 2021; e106540. doi:10.15252/embj.2020106540

55. Lucas-Hourani M, Dauzonne D, Munier-Lehmann H, Khiar S, Nisole S, Dairou J, et al. Original Chemical Series of Pyrimidine Biosynthesis Inhibitors That Boost the Antiviral Interferon Response. Antimicrob Agents Chemother. 2017;61. doi:10.1128/AAC.00383-17 
A

NI
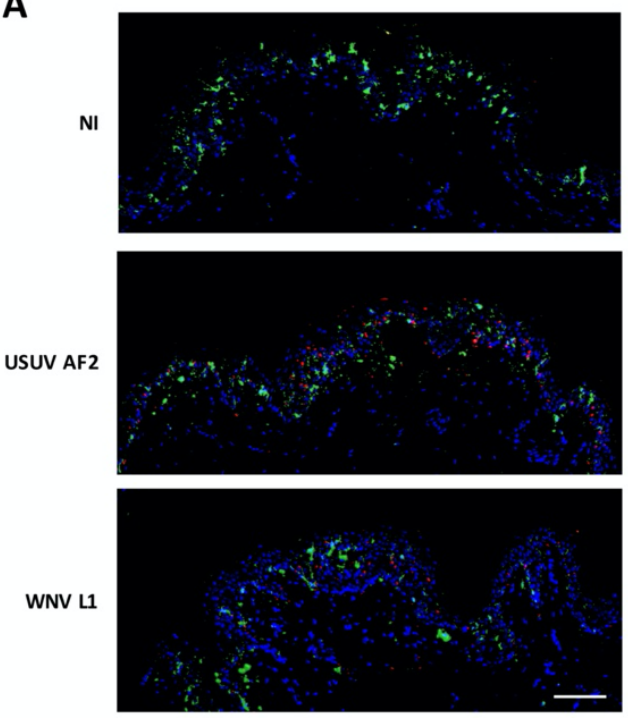

C

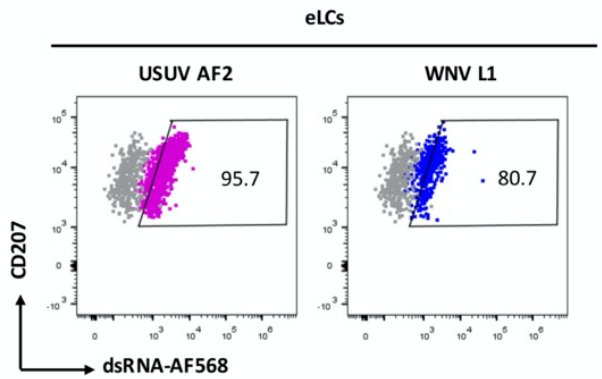

D

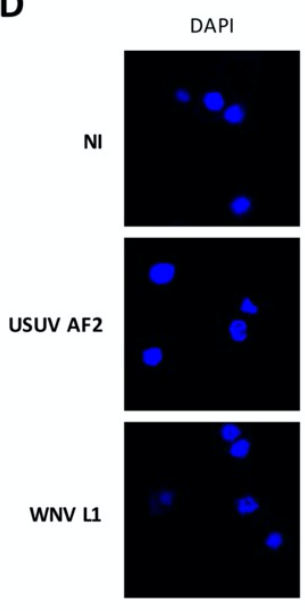

dsRNA
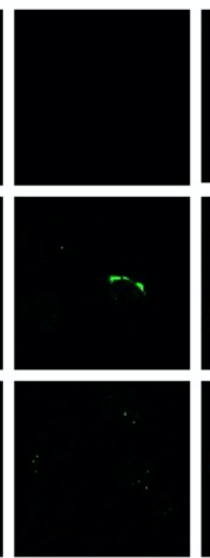

Langerin

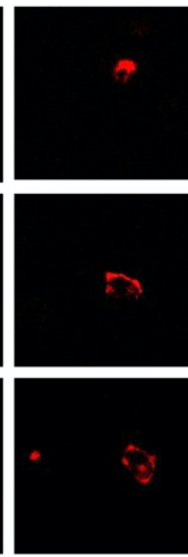

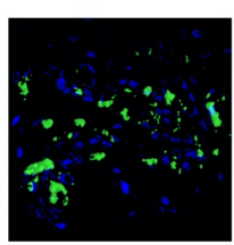
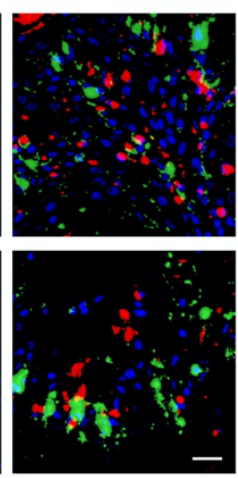

B

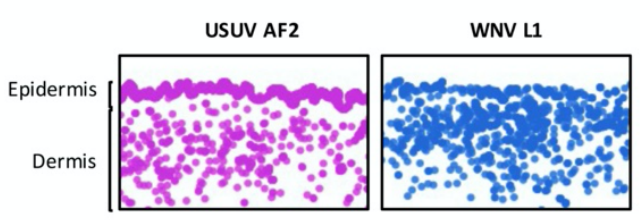

$\mathrm{CD} 1 \mathrm{a}+$ / viral protein E+ cells

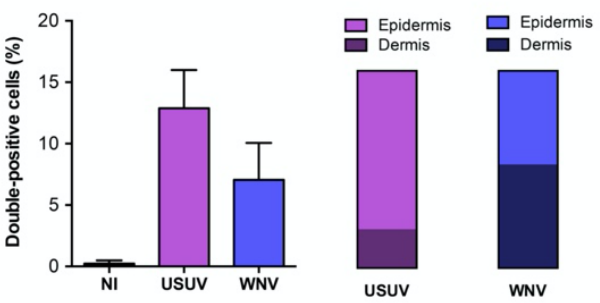

Figure 1. Unlike WNV, USUV infects preferentially Langerhans cells in human skin.

(A) Human skin explants were left uninfected (NI) or infected by USUV AF2, WNV L1 at MOI 1. $24 \mathrm{hpi}$, tissues were fixed and paraffin-embedded, and analyzed by multiplex immunofluorescent assay using a pan-flavivirus anti-viral envelope E antibody (red) and an anti-CD1a antibody (green). Nuclei were stained with DAPI (blue). Images were acquired on a confocal scanner. Scale bars: $100 \mu \mathrm{m}$ (left) or $20 \mu \mathrm{m}$ (right).

(B) Pan-flavivirus (red) and CD1a (green) staining from (A) was quantified using the image analysis platform Halo. Screenshots of double positive cells (infected CD1a+ cells) in whole sections are presented (top). Quantification of double positive cells in each condition was represented as mean \pm SD (bottom left), as well as the distribution of double positive cells between the epidermis and the dermis (bottom right).

(C) Epidermal cells were purified from skin explants and infected with USUV AF2, WNV L1 at MOI 2. At 48 hpi, viral replication was estimated by flow cytometry by staining intracellular viral dsRNA in CD207+ Langerhans cells (eLCs) or CD207- epidermal cells (mainly keratinocytes).

(D) Epidermal cells were purified from skin explants and infected with USUV AF2, WNV L1 at MOI 2. At 24 hpi, cells were fixed and stained for nuclei (blue), dsRNA (green) and CD207 (red). Images were acquired on a Leica SP5-SMD microscope. Scale bar: $10 \mu \mathrm{m}$.

(E) Epidermal CD1a+ cells were infected with USUV AF2, WNV L1 at MOI 2 for $24 \mathrm{~h}$ or $48 \mathrm{~h}$. Intracellular viral RNA was quantified by RT-qPCR. Results from 3 independent experiments performed in duplicate are shown. 
A
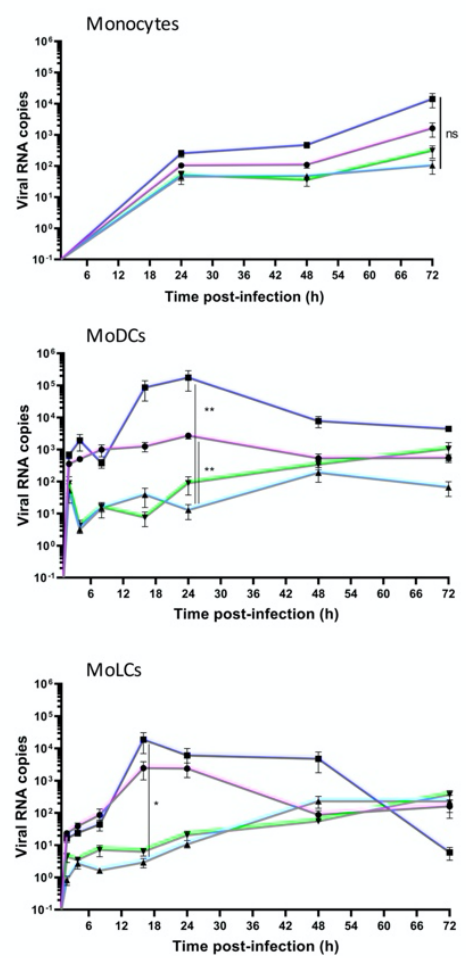

- USUV AF2

- usuv eu2

$\star$ WNV L1

$\rightarrow$ WNV L2

B

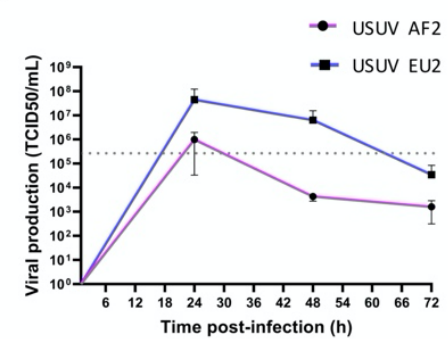

- WNV L1

$\rightarrow$ WNV L2

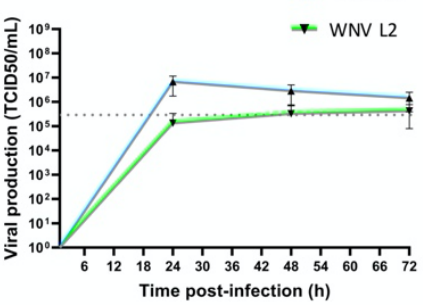

C

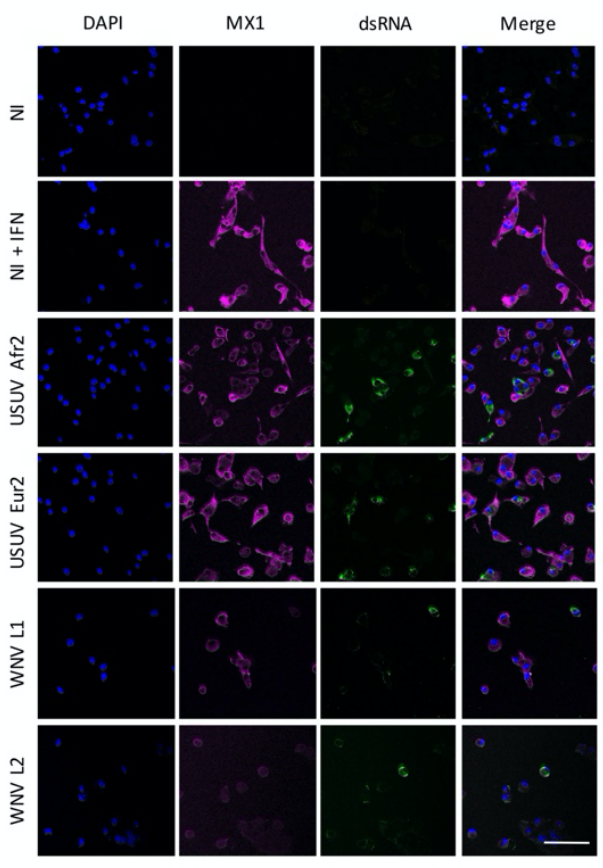

Figure 2. Viral replication of WNV and USUV in primary human myeloid cells.

(A) Human monocytes and autologous MoDCs or MoLCs were infected by USUV AF2, USUV EU2, WNV L1 or WNV L2 at MOI 1. Cells were harvested at indicated times post-infection and intracellular viral RNA was quantified by RT-qPCR. Data represent 3 independent experiments run in technical duplicate, represented as mean \pm SEM and shown in intracellular viral RNA copies. ${ }^{*} p<0.05,{ }^{* *} p<0.01$ have been determined using a Mann-Whitney $t$-test relative to indicated condition.

(B) MoLCs were infected by USUV AF2, USUV EU2, WNV L1 or WNV L2 at MOI 1. Viral production in MoLCs was assessed by TCID50/ml titration of supernatants at indicated times on Vero cells. The dotted line shows the titer of the viral inoculum. Results are represented as mean \pm SD of 3 independent experiments performed in duplicate.

(C) MoLCs were infected at MOI 1 by USUV AF2, USUV EU2, WNV L1, WNV L2, not infected (NI) or pretreated by IFN- $\alpha 2$ (NI + IFN). Cells were fixed at 24hpi (USUV) or $48 \mathrm{hpi}$ (WNV) and stained for nuclei (blue), dsRNA (green) and MX1 (purple). Images were acquired on a Leica SP5-SMD microscope. Scale bar: $35 \mu \mathrm{m}$. 
A
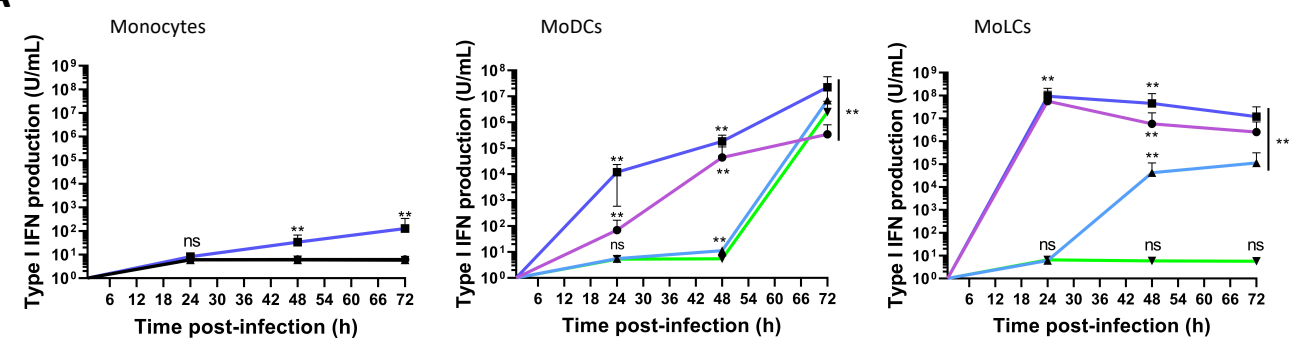

B
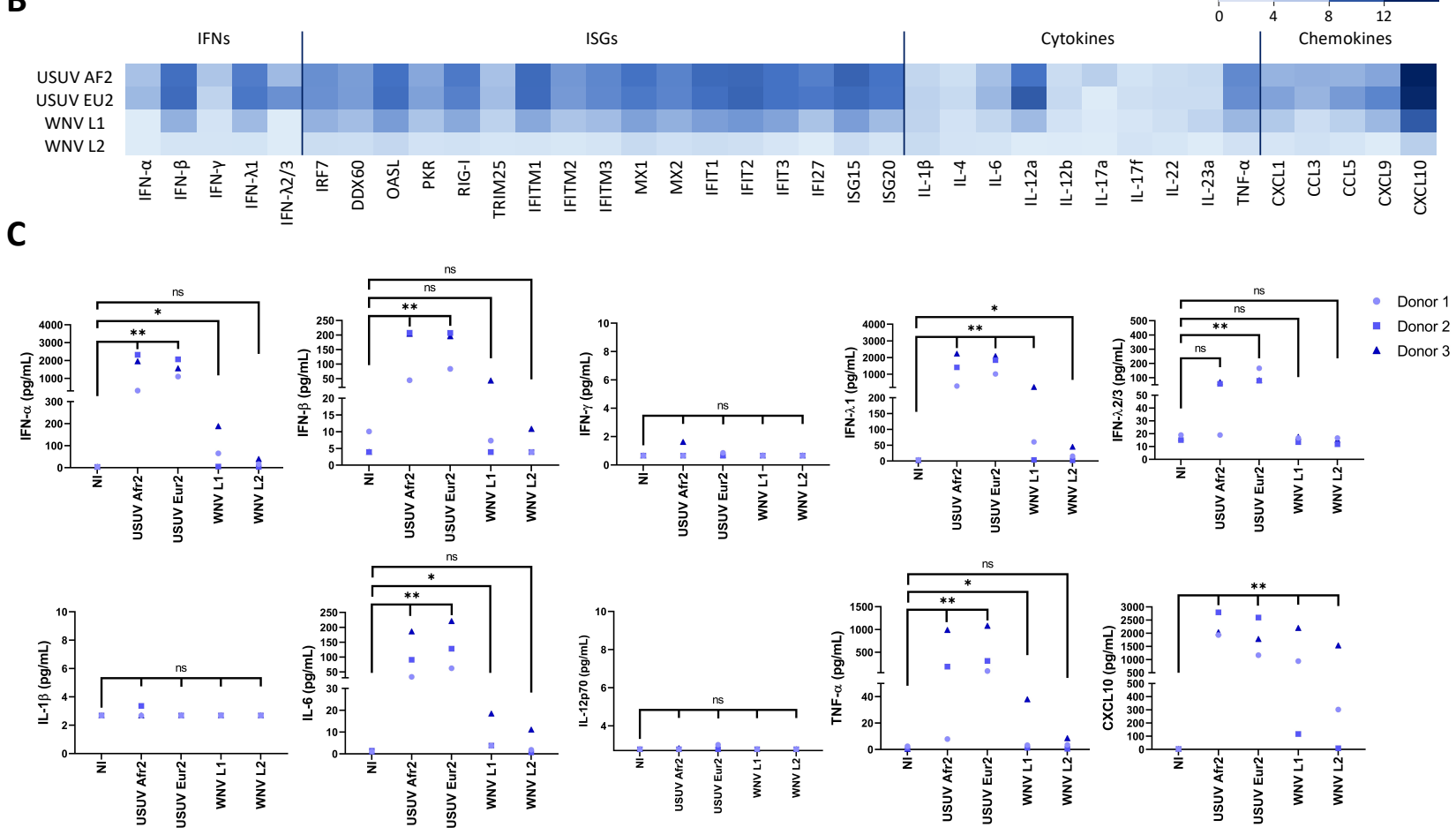

Figure 3. Characterization of USUV- and WNV-triggered innate immune response in MoLCs.

(A) Human monocytes and autologous MoDCs or MoLCs were infected with USUV AF2, USUV EU2, WNV L1 or WNV L2 at MOI 1 for 24, 48 or 72 hours. Type I IFN secreted in the culture medium by infected cells was quantified on STING-37 reporter cells. Data are from 3 independent experiments performed in duplicate and are represented as mean $\pm S D$. ${ }^{*} p<0.05,{ }^{* *} p<0.01$ have been determined using a Mann-Whitney $t$-test relative to control cells. ns $=$ non-significant.

(B) MoLCs were harvested at $24 \mathrm{hpi}$ and transcripts of a panel of proteins including IFNs, ISGs, cytokines and chemokines were quantified by RT-qPCR. Results are summarized on a heatmap showing low (light blue) to high (dark blue) transcription intensity. Data represent the mean of the log2 transcription intensity of each indicated transcript, from four independent experiments run in technical duplicate, relative to control cells (NI). (C) Cytokine concentrations in supernatants from infected MoLCs were measured using the multiplex beadbased immunoassay LEGENDplex (BioLegend) at $48 \mathrm{hpi}$. Data are from 3 independent experiments performed on cells from 3 donors. ${ }^{*} p<0.05,{ }^{* *} p<0.01$ have been determined using a Mann-Whitney $t$-test relative to uninfected cells $(\mathrm{NI}) . \mathrm{ns}=$ non-significant. 
A

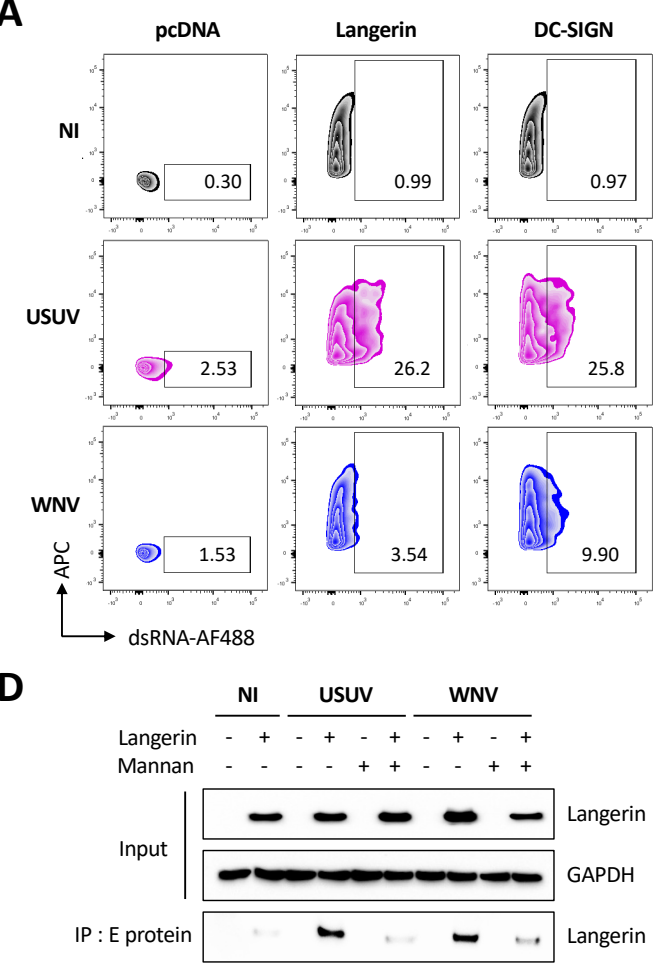

$\mathbf{E}$

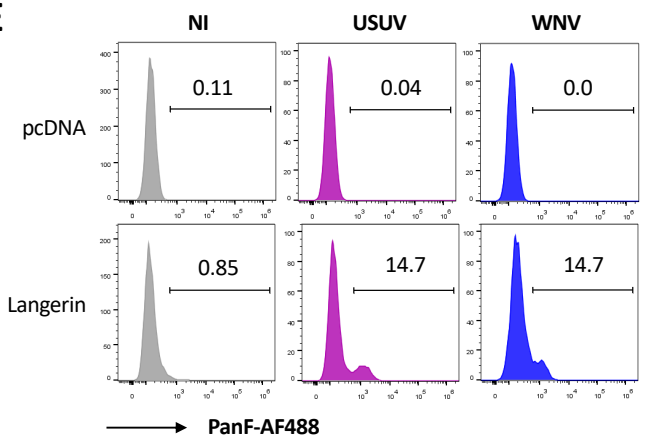

B
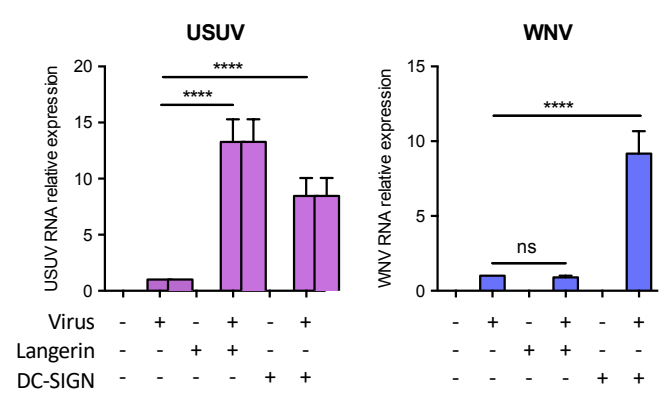

C
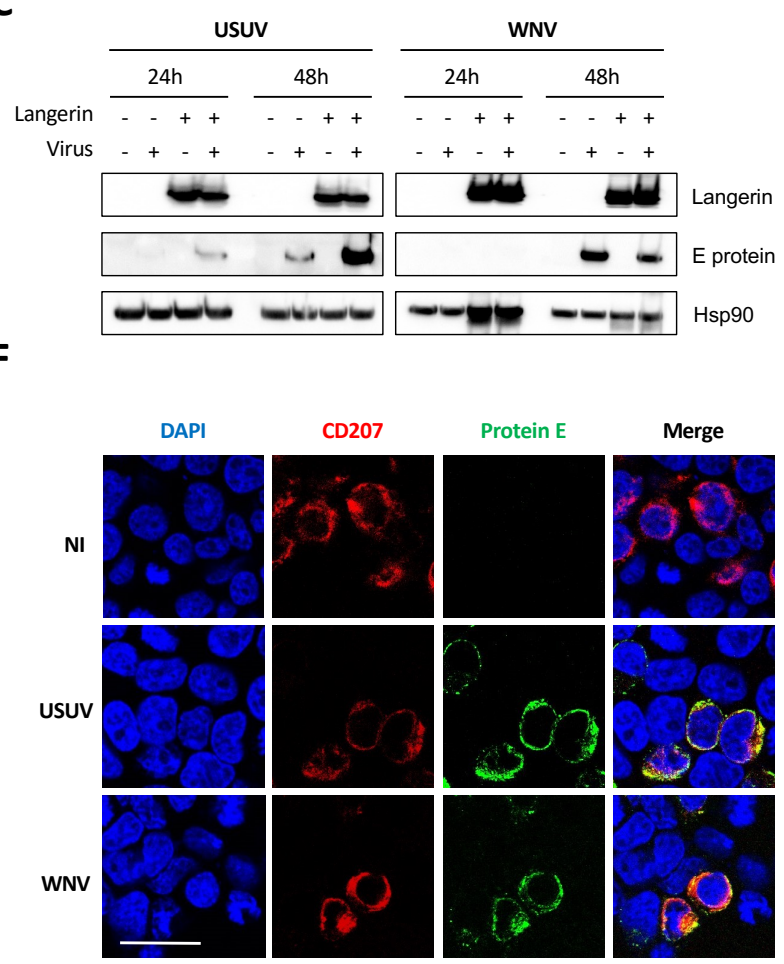

Figure 4. Langerin expression allows USUV but not WNV replication in HEK293T cells.

(A and B) HEK293T cells were transfected with an empty plasmid (pcDNA) or with plasmids encoding langerin or DC-SIGN. 24h post transfection, cells were infected with USUV AF2 and WNV L1 at MOI 0.5 for $48 \mathrm{~h}$, or left non-infected (NI). (A) USUV and WNV replication was assessed by flow cytometry following intracellular immunostaining using anti-dsRNA and Alexa Fluor 488, anti-langerin-APC and anti-DC-SIGN-APC antibodies. The percentage of infected cells among langerin-positive and DC-SIGN-positive (transfected) cells is shown. Results are from one representative experiment of 3 independent experiment.

(B) Same as (A), except that viral replication was estimated by quantifying viral RNA by RT-qPCR. Results are represented as mean fold change \pm SD of 3 independent experiments performed in duplicate, relative to infected control cells (transfected with pcDNA). ${ }^{* * *} \mathrm{p}<0.0001$, as determined by Student's t-test. ns $=$ nonsignificant.

(C) HEK293T cells were transfected with an empty plasmid (-) or with a plasmid encoding human langerin (+), as indicated. At $24 \mathrm{~h}$ post transfection, cells were infected with USUV AF2 or WNV L1 at MOI 0.5 for $24 \mathrm{~h}$ or $48 \mathrm{~h}$, or left uninfected. Cell lysates were subjected to western-blotting using anti-langerin or pan-flavivirus antibodies.

(D) HEK293T cells were transfected or not with a plasmid encoding langerin. At $24 \mathrm{~h}$ post transfection, cells were pretreated or not with $1 \mathrm{mg} / \mathrm{ml}$ mannan for $1 \mathrm{~h}$ and infected with USUV AF2 and WNV L1 at MOI 5 for 30 min, or left noninfected $(\mathrm{NI})$. Following cell lysis, whole-cell lysates were subjected to IP with anti-E protein antibody followed by Western blot analysis with the anti-langerin antibody.

(E) HEK293T cells were transfected with an empty plasmid (pcDNA) or with a plasmid encoding langerin. At $24 \mathrm{~h}$ post transfection, cells were infected with USUV AF2 and WNV L1 at MOI 5 for 30 min, or left noninfected (NI). Cells were fixed and subjected to flow cytometry following surface immunostaining the viral $E$ protein with the pan-flavirus anti-E antibody. The percentage of cells expressing the viral $E$ protein is shown. Results are from one representative experiment of 2 independent experiments.

(F) HEK293T cells expressing langerin were left uninfected (NI) or infected by USUV AF2 or WNV L1 at MOI 5 for $30 \mathrm{~min}$. Cells were fixed and stained for nuclei (blue), langerin (red) and $\mathrm{E}$ protein (green). Images were acquired on a Leica SP5-SMD microscope. Scale bar: $15 \mu \mathrm{m}$. 
A

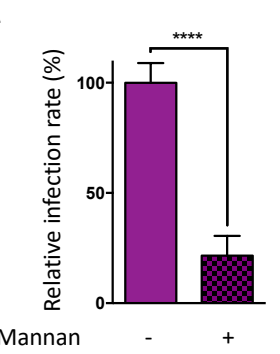

$\mathbf{E}$

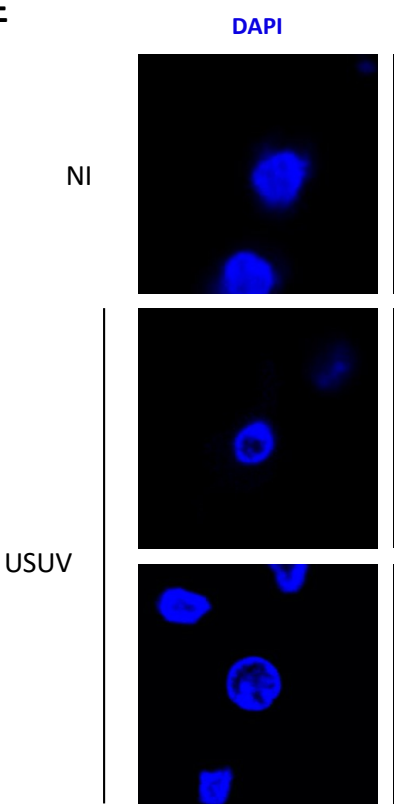

B
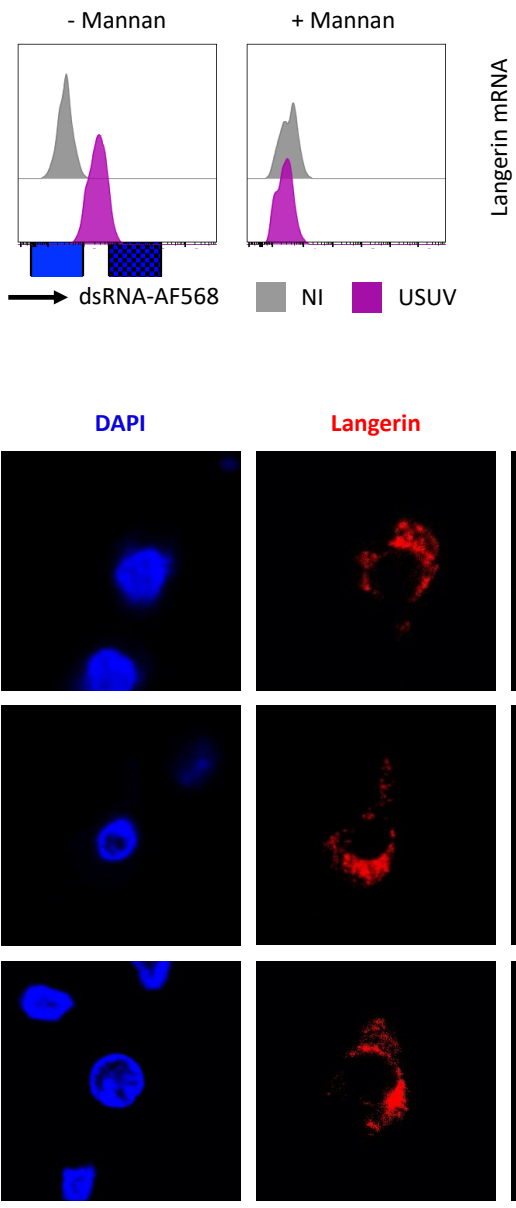

C
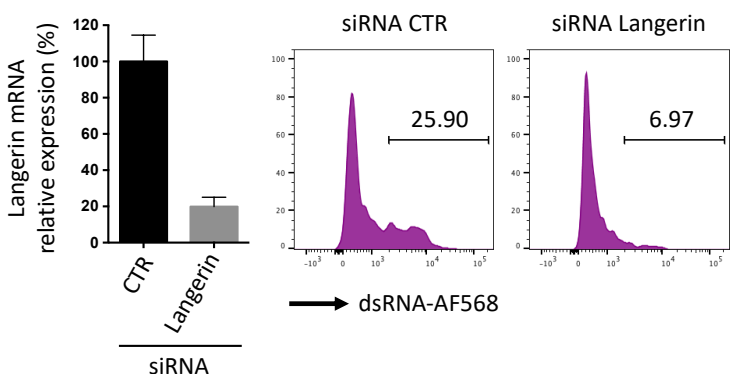

E protein
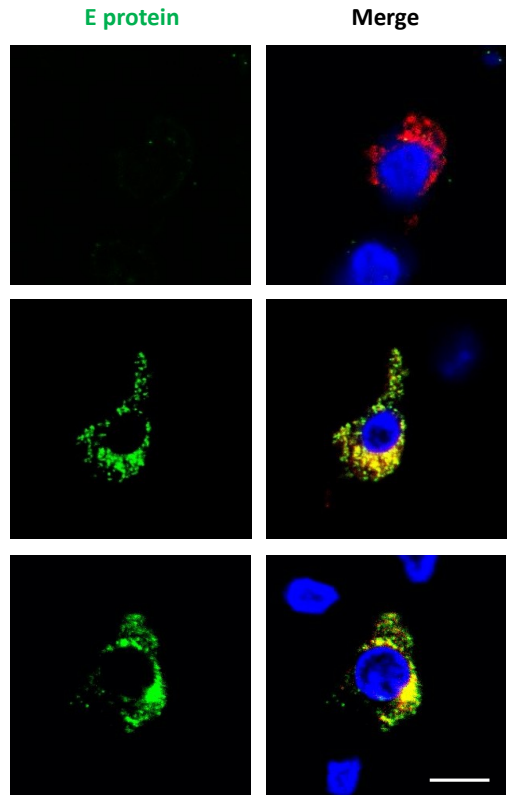

Figure 5. Langerin allows USUV entry and replication in human LCs.

(A) MoLCs were left untreated or treated for $1 \mathrm{~h}$ with $1 \mathrm{mg} / \mathrm{ml}$ mannan and infected with USUV AF2 at MOI 2 for $24 \mathrm{~h}$. Viral replication was assessed by quantifying the amount of viral RNA in cells by RT-qPCR. Data represent the mean \pm SD of 3 independent experiments performed in triplicate on cells from 3 different donors. (B) Epidermal LCs purified from human skin were treated or not with $1 \mathrm{mg} / \mathrm{ml}$ mannan for $1 \mathrm{~h}$ and infected with USUV AF2 at MOI 2. At $24 \mathrm{hpi}$, viral replication was estimated by flow cytometry using dsRNA and Alexa Fluor 568 antibodies. Data are from one representative experiment of two independent experiments.

(C and D) Epidermal LCs purified from human skin were transfected with siRNA control (CTR) or siRNA targeting langerin. Relative expression of langerin was estimated by RT-qPCR analysis (C). Cells were infected with USUV AF2 for $24 \mathrm{~h}$ at MOI 2 and viral replication was assessed by flow cytometry using antidsRNA and AF568 antibodies (D). Data are from one representative experiment of two independent experiments.

(E) Epidermal LCs purified from skin explants were infected (USUV) or not (NI) with USUV AF2 for 30 min at MOI 5. Cells were fixed and stained for nuclei (blue), E protein (green) and CD207 (red). Images were acquired on a Leica SP5-SMD microscope. Scale bar: $20 \mu \mathrm{m}$. 
bioRxiv preprint doi: https://doi.org/10.1101/2021.08.17.456611; this version posted November 13, 2021. The copyright holder for this preprint (which was not certified by peer review) is the author/funder, who has granted bioRxiv a license to display the preprint in perpetuity. It is made available under aCC-BY-NC-ND 4.0 International license.

A

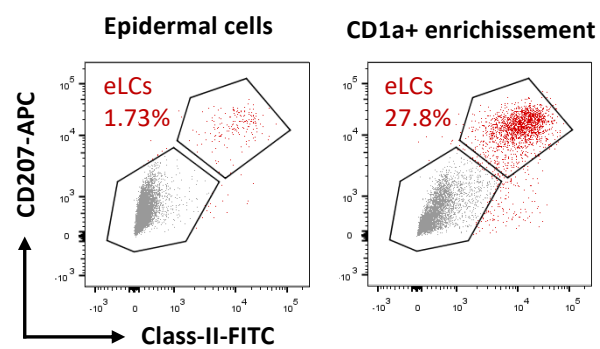

B
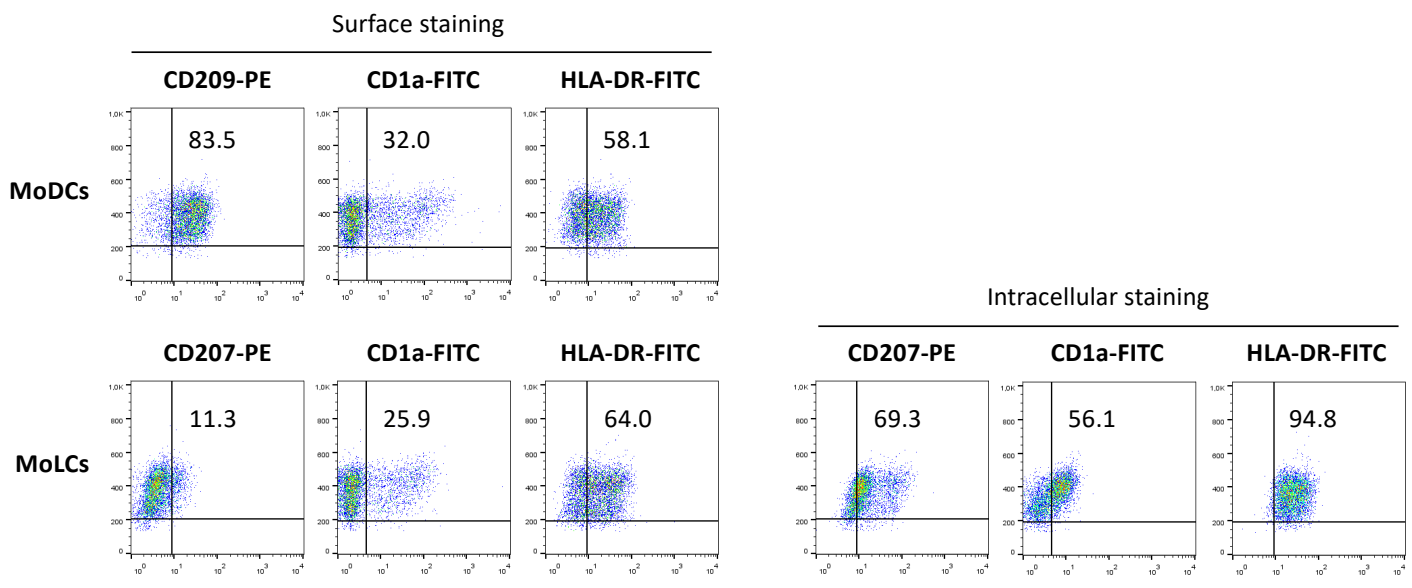

Figure S1. Phenotyping of eLCs, MoDCs and MoLCs.

(A) Expression of CD207 and HLA-DR (class II) was assessed by flow cytometry using anti-CD207 and antiHLA-DR antibodies, respectively. Staining was performed on both total epidermal cells (left panel) and following enrichment of CD1a+ cells (right panel). eLCs are colored in red and epidermal cells appear in grey. A representative phenotyping is shown.

(B) Expression of CD209 (DC-SIGN), CD207 (Langerin), CD1a and HLA-DR (class II) was assessed by flow cytometry using appropriate antibodies. MoDCs were subjected to surface staining only (top panel) and MoLCs to both surface (bottom left panel) and intracellular (bottom right panel) staining. Positive cells for each marker appear in the top right quarter of each dot-plot. Representative phenotypes are shown. 

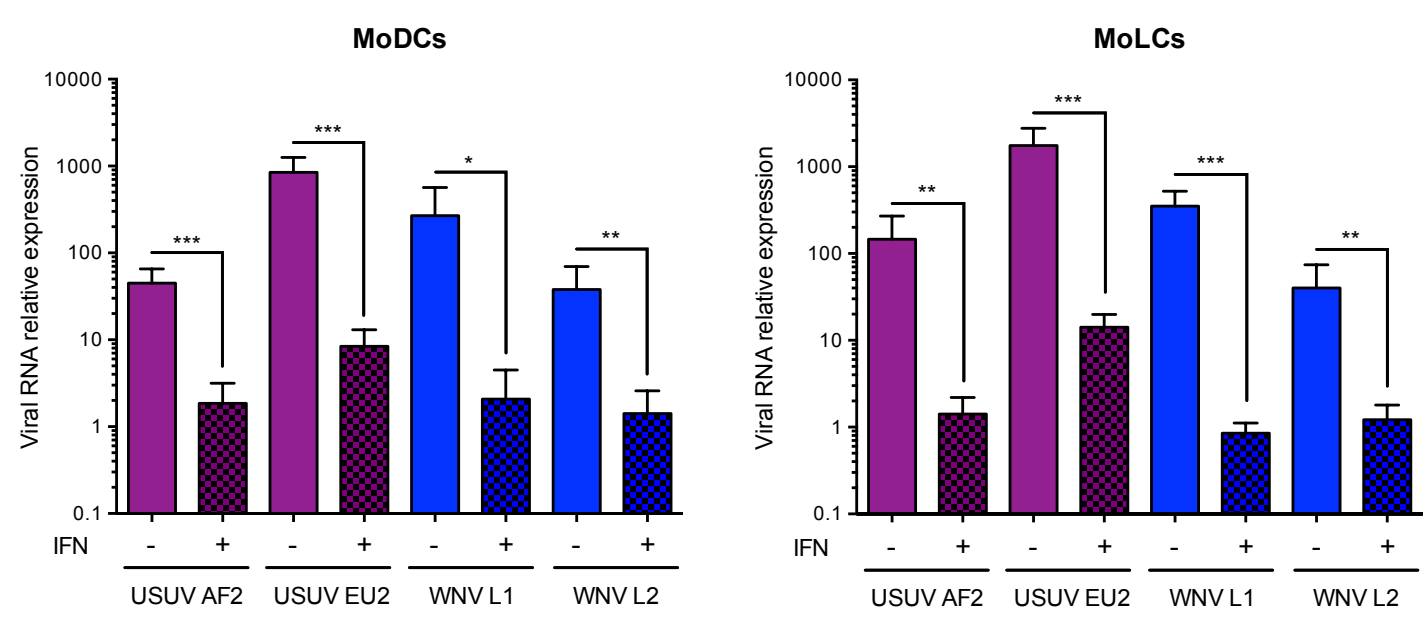

Figure S2. IFN- $\alpha 2$ inhibits USUV and WNV replication in MoDCs and MoLCs.

MoDCs or MoLCs were pre-treated or not with $1000 \mathrm{U} / \mathrm{mL}$ of IFN-alpha2 for $24 \mathrm{~h}$ before infection with USUV AF2, USUV EU2, WNV L1 or WNV L2 at MOI 1. At $24 \mathrm{hpi}$, total RNA were extracted and viral RNA was quantified by RT-qPCR. Results from 3 independent experiments performed in duplicate are shown. ${ }^{* *} p<$ $0.001,{ }^{* *} p<0.01,{ }^{*} p<0.05$, as determined by Student's t-test. 
A
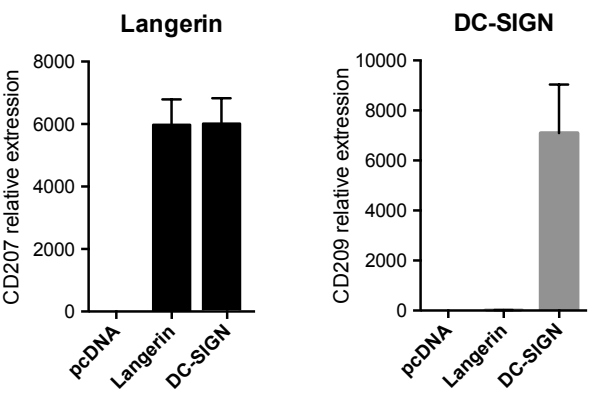

B
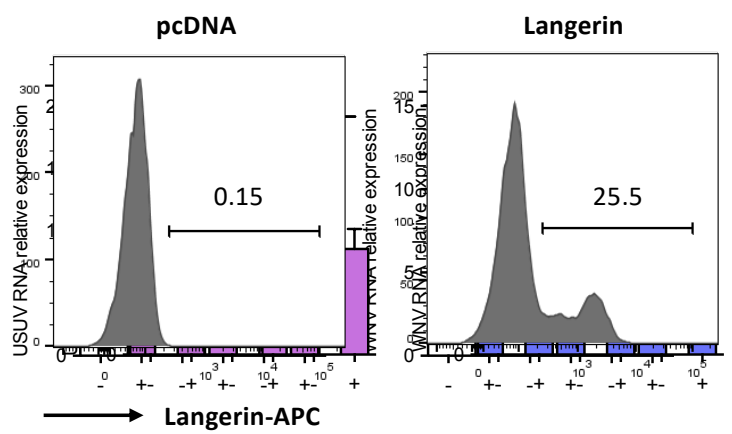

Figure S3. Expression levels of Langerin and DC-SIGN in HEK293T cells.

(A) In parallel of the experiments shown in Figures 4A and 4B, the expression of Langerin and DC-SIGN in HEK293T cells was estimated at $24 \mathrm{~h}$ post-transfection by RT-qPCR. Relative expression of Langerin and DCSIGN mRNA expression is represented as mean values \pm SD.

(B) The expression of Langerin in transfected HEK293T cells used in the experiment shown in Figure 4D and 4E was evaluated by flow cytometry using an anti-langerin antibody. The percentage of Langerin-expressing cells in indicated. 